\title{
Estación Biológica Senda Darwin: Investigación ecológica de largo plazo en la interfase ciencia-sociedad
}

\author{
Senda Darwin Biological Station: Long-term ecological research at the interface between \\ science and society
}

\author{
MARTÍN R. CARMONA ${ }^{1,2,5}$, J. C. ARAVENA ${ }^{6}$, MARCELA A. BUSTAMANTE-SÁNCHEZ1,2 , JUAN L. CELIS-DIEZ1,2 \\ ANDRÉS CHARRIER ${ }^{2}$, IVÁN A. DÍAZ ${ }^{8}$, JAVIERA DÍAZ-FORESTIER ${ }^{1}$, MARÍA F. DÍAZ ${ }^{1,}$, ${ }^{2}$, AURORA GAXIOLA ${ }^{1,2,5}$,

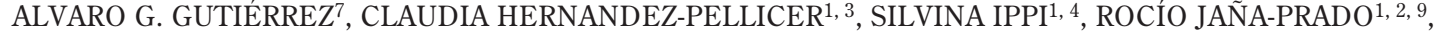 \\ PAOLA JARA-ARANCIO ${ }^{1,4}$, JAIME JIMENEZ ${ }^{13}$, DANIELA MANUSCHEVICH ${ }^{1,2}$, PABLO NECOCHEA ${ }^{11}$, MARIELA \\ NUÑEZ-AVILA ${ }^{1,2,8}$, CLAUDIA PAPIC ${ }^{11}$, CECILIA PÉREZ ${ }^{2}$, FERNANDA PÉREZ ${ }^{1,2,5}$, SHARON REID ${ }^{1,2}$, LEONORA \\ ROJAS $^{1}$, BEATRIZ SALGADO ${ }^{1,2}$, CECILIA SMITH-RAMÍREZ ${ }^{1,2}$, ANDREA TRONCOSO ${ }^{12}$, RODRIGO A. \\ VÁSQUEZ1, ${ }^{1,}$, MARY F. WILLSON ${ }^{1}$, RICARDO ROZZI ${ }^{1} \&$ JUAN J. ARMESTO $^{1,2,5, \text { * }}$ \\ ${ }^{1}$ Instituto de Ecología y Biodiversidad (IEB), Facultad de Ciencias, Universidad de Chile, Las Palmeras 3425, \\ Ñuñoa, Casilla 653, Santiago, Chile \\ ${ }^{2}$ Centro de Estudios Avanzados en Ecología y Biodiversidad (CASEB), Departamento de Ecología \\ Pontificia Universidad Católica de Chile, Alameda 340, Casilla 114-D, Santiago, Chile, 833-1150 \\ ${ }^{3}$ Centro de Estudios Avanzados en Zonas Áridas (CEAZA), Casilla 599 - Raúl Bitrán s/n, Colina El Pino, La Serena, Chile \\ ${ }^{4}$ Departamento de Ciencias Ecológicas, Facultad de Ciencias, Universidad de Chile, Las Palmeras 3425, \\ Ñuñoa, Casilla 653, Santiago, Chile \\ ${ }^{5}$ Laboratorio Internacional de Cambio Global (LINCGlobal), UC-CSIC, Departamento de Ecología \\ Pontificia Universidad Católica de Chile, Alameda 340, Casilla 114-D, Santiago, Chile, 833-1150 \\ ${ }^{6}$ Centro de Estudios del Quaternario (CEQUA), Avenida Bulnes 01890, Casilla 737, Punta Arenas, Chile \\ ${ }^{7}$ Department of Ecological Modelling, Helmholtz Centre for Environmental Research (UFZ), \\ Permoserstr. 15, 04318 Leipzig, Germany \\ ${ }^{8}$ Instituto de Silvicultura, Facultad de Ciencias Forestales y Recursos Naturales, Universidad Austral de Chile, \\ Campus Isla Teja s/n, Casilla 567, Valdivia, Chile \\ ${ }^{9}$ School of Biological Sciences, University of Canterbury, Private Bag 4800, Christchurch 8140, New Zealand \\ 10 Departamento de Ciencias Biológicas, Facultad de Ciencias Biológicas, Universidad Andrés Bello, Santiago, Chile \\ ${ }^{11}$ PricewaterhouseCoopers, Av. Andrés Bello 2711 Piso 3, Las Condes, Santiago, Chile \\ 12 Comisión Nacional de Ciencia y Tecnología, CONICYT, Canadá 308, Providencia, Santiago, Chile \\ ${ }^{13}$ Laboratorio de Vida Silvestre, Universidad de Los Lagos, Fuchslocher 1305, Casilla 933, Osorno, Chile \\ *Autor correspondiente: jarmesto@bio.puc.cl
}

\section{RESUMEN}

La Estación Biológica Senda Darwin (EBSD) constituye un centro de investigación inmerso en el paisaje rural del norte de la Isla de Chiloé $\left(42^{\circ} \mathrm{S}\right)$, donde fragmentos del bosque siempreverde original coexisten con praderas de uso ganadero, turberas de Sphagnum, matorrales sucesionales, plantaciones de Eucalyptus y otras formaciones de origen antropogénico. Desde 1994 hemos realizado estudios de largo plazo centrados en algunas especies de plantas (e.g., Pilgerodendron uviferum D. Don) y animales (e.g., Aphrastura spinicauda Gmelin, Dromiciops gliroides [Thomas]) catalogados como amenazados o escasamente conocidos y en ecosistemas nativos de importancia regional y global (e.g., turberas de Sphagnum, bosque Valdiviano y Nordpatagónico). Las investigaciones han considerado las respuestas de las especies y de los ecosistemas frente al cambio antropogénico del paisaje y cambio climático, así como los efectos de diferentes formas de manejo. Este escenario es semejante al de otras regiones de Chile y Latinoamérica lo que da generalidad a nuestros resultados y modelos. En este período, investigadores asociados a la EBSD han producido más de un centenar de publicaciones en revistas nacionales e internacionales y 30 tesis de pre y postgrado. Entendiendo el papel clave de los seres humanos en los procesos ecológicos de la zona rural, la EBSD ha desarrollado un programa de educación ecológica y vinculación del avance científico con la sociedad local y nacional. La integración de la EBSD a la naciente red de Sitios de Estudios Socio-Ecológicos de Largo Plazo en Chile consolidará y fortalecerá la investigación básica y aplicada que realizamos para proyectarla hacia la siguiente década.

Palabras clave: área protegida privada, biodiversidad, bosques templados, educación, investigación socioecológica de largo plazo. 


\begin{abstract}
Senda Darwin Biological Station (SDBS) is a field research center immersed in the rural landscape of northern Chiloé island $\left(42^{\circ} \mathrm{S}\right)$, where remnant patches of the original evergreen forests coexist with open pastures, secondary successional shrublands, Sphagnum bogs, Eucalyptus plantations and other anthropogenic cover types, constituting an agricultural frontier similar to other regions in Chile and Latin America. Since 1994, we have conducted long-term research on selected species of plants (e.g., Pilgerodendron uviferum) and animals (e.g., Aphrastura spinicauda, Dromiciops glirioides) that are considered threatened, poorly known or important for their ecological functions in local ecosystems, and on ecosystems of regional and global relevance (e.g., Sphagnum bogs, North Patagonian and Valdivian rain forests). Research has assessed the responses of species and ecosystems to anthropogenic land-use change, climate change, and the impact of management. During this period, more than 100 scientific publications in national and international journals, and 30 theses (graduate and undergraduate) have been produced by scientists and students associated with SDBS. Because of our understanding of the key role that humans play in ecological processes at this agricultural frontier, since the establishment of SDBS we have been committed to creative research on the communication of science to society and ecological education. The integration of SDBS to the nascent Chilean network of long-term socio-ecological research will consolidate and strengthen basic and applied research to project our work into the next decade.
\end{abstract}

Key words: biodiversity, education, long-term socio-ecological research, private protected area, temperate rainforests.

\section{INTRODUCCIÓN}

Los bosques templados del sur de Sudamérica, distribuidos entre $\operatorname{los} 35^{\circ}$ y $55^{\circ} \mathrm{S}$ en el margen occidental del continente, se caracterizan por el alto grado de endemismo de muchos taxa, entre los cuales se encuentran relictos de antiguas biotas ya desaparecidas (Armesto et al. 1996, Villagrán \& Hinojosa 1997). Aproximadamente en el punto medio del rango latitudinal de estos bosques, en el extremo norte de la Isla Grande de Chiloé, se encuentra la Estación Biológica Senda Darwin ( $42^{\circ} 53^{\prime} \mathrm{S}$, $73^{\circ} 40^{\prime} \mathrm{O}$ ), en adelante EBSD.

Esta zona presenta al menos tres características de interés para estudios ecológicos con un foco en el funcionamiento y servicios de los ecosistemas: (1) Aquí los glaciares alcanzaron su límite noroccidental durante el último período glacial (Villagrán et al. 1995), lo que implica que los bosques actuales colonizaron dicha zona hace aproximadamente 20 mil años, desplazando formaciones de turberas y matorrales, siguiendo el calentamiento postglacial. Así, los bosques mantienen un grado de resiliencia frente a grandes perturbaciones y cambios climáticos pasados, que nos puede informar sobre sus potenciales respuestas a cambios futuros. (2) Actualmente coexisten aquí los dos tipos forestales nativos más importantes y con mayor diversidad biológica del bosque siempreverde lluvioso, el bosque Valdiviano y Nordpatagónico, ambos asociados a especies emblemáticas de plantas y animales que forman parte del patrimonio natural de la región. El conocimiento de la dinámica y las formas de manejo sustentable de estos bosques es aún limitado (Gutiérrez et al. 2009). (3) Es una frontera agrícola-forestal (Jaña-Prado et al. 2006), donde el proceso de cambio de uso del suelo está ocurriendo con gran rapidez (Echeverría et al. 2007), asociado a la fragmentación y conversión de los bosques nativos en otros tipos de cobertura "antropogénica" tales como praderas, plantaciones de eucaliptos, turberas de Sphagnum (pomponales), y matorrales. Estos cambios están produciendo efectos biológicos y culturales que estamos recién comenzando a comprender.

La EBSD contiene una muestra representativa del mosaico de comunidades que caracteriza esta zona (Fig. 1, Apendice), brindando la oportunidad de investigar y comprender el funcionamiento de los ecosistemas en un paisaje rural semejante a otras regiones del sur de Chile y Sudamérica (Armesto et al. 1998), y entender los cambios biológicos y culturales que modelarán el paisaje en el futuro. Durante más de 15 años, la EBSD ha apoyado la investigación científica de punta sobre biodiversidad y procesos ecosistémicos que se ha llevado a cabo en este ambiente rural. En este período, con el auspicio y apoyo de EBSD, se han generado más de un centenar de publicaciones científicas, en revistas nacionales e 
internacionales, y 30 tesis de pre y postgrado (Fig. 2). Las investigaciones se han centrado en un grupo ecológicamente relevante de especies de plantas y animales del bosque (e.g., especies amenazadas, mutualistas, dominantes, funcionalmente claves, etc.) y en su contexto ecosistémico. Muchas investigaciones se extienden más allá de los tres años que caracterizan la mayoría de los estudios ecológicos en Chile, por lo que se definen como estudios de largo plazo (Armesto 1990). El objetivo de este artículo es presentar una síntesis de los avances y proyecciones de las principales líneas de trabajo de la EBSD, especialmente aquellas de largo plazo - tanto en investigación como en educación y difusión de la ciencia. Recientemente, la EBSD se ha integrado a la naciente Red Chilena de Estudios Socio-Ecológicos de Largo Plazo (ESELP), cuya organización, objetivos y agenda son presentadas en esta sección temática especial de la Revista Chilena de Historia Natural.

\section{Estación Biológica Senda Darwin Chiloé, X Region, Chile}

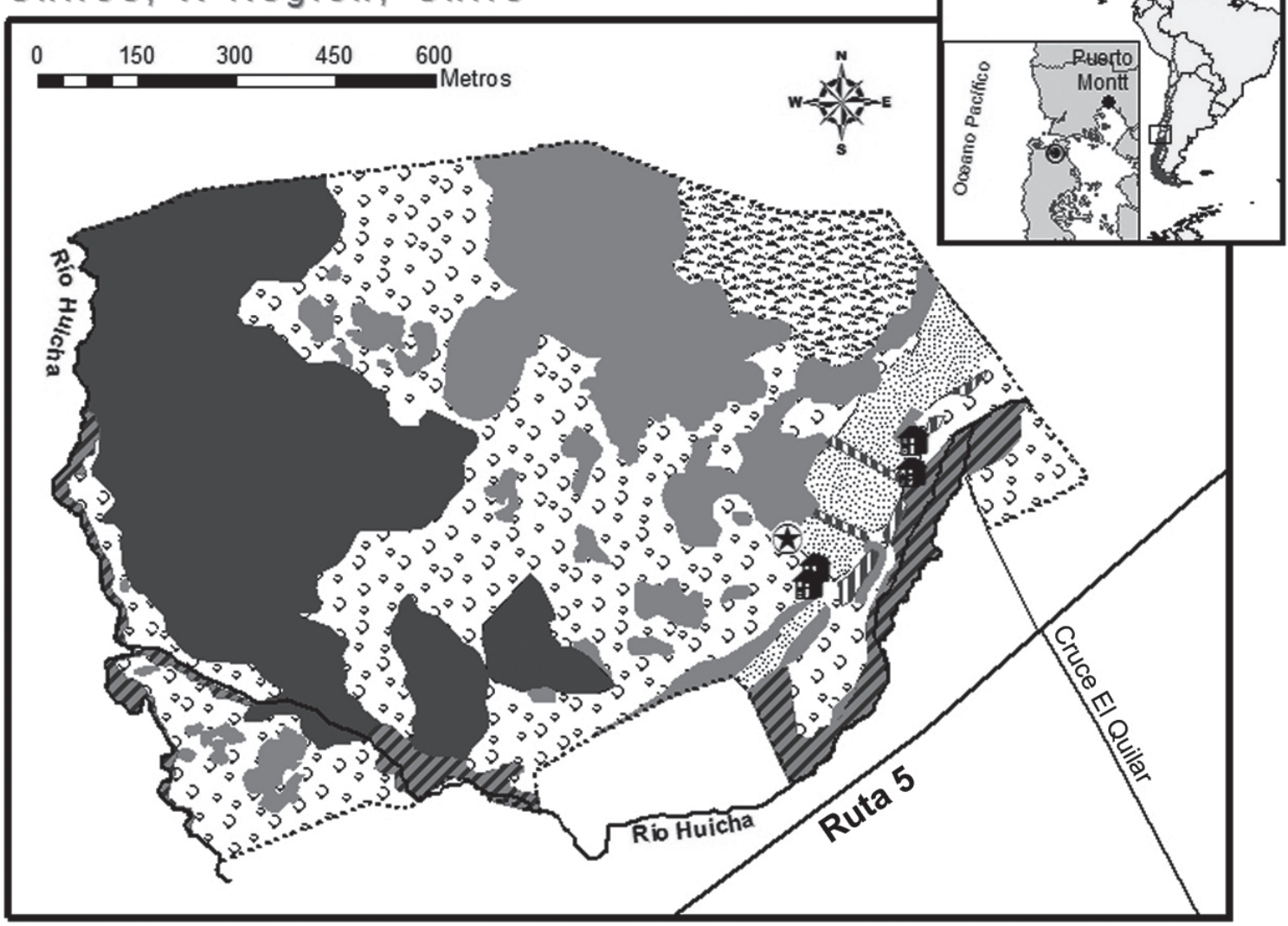

\section{Leyenda}

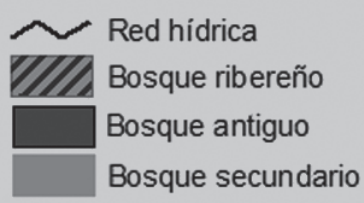

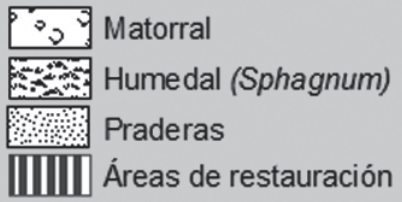

Fig. 1: Mapa de la Estación Biológica Senda Darwin (EBSD), distinguiendo los principales tipos de vegetación del paisaje rural, y parte de la infraestructura presente. Coordenadas geográficas (Estación climatológica): $42^{\circ} 53^{\prime}$ S, $73^{\circ} 39^{\prime}$ W. Superficie total: 115 ha.

Map of Senda Darwin Biological Station (EBSD), showing the main vegetation types of the rural landscape, and part of the station's facilities. Geographic coordinates (Weather station): $42^{\circ} 53^{\prime} \mathrm{S}, 73^{\circ} 39^{\prime} \mathrm{W}$. Total surface: 115 ha. 


\section{PROGRAMA DE INVESTIGACION}

\section{Caracterización climática}

La caracterización del clima local, sus fluctuaciones estacionales e interanuales, así como las tendencias de largo plazo, representan un componente esencial para todo estudio de ecosistemas terrestres. La EBSD cuenta desde 1997 con un registro continuo (con resolución temporal de una hora) de la dirección y velocidad del viento, radiación solar global, temperatura del aire y suelo, humedad relativa del aire y precipitación. Este es el único registro climático instrumental vigente en la zona norte de Chiloé y constituye un referente para los estudios ecológicos en la zona. El resumen de los registros mensuales de temperatura y precipitación (Fig. 3, Tabla 1) permite caracterizar los patrones de estacionalidad de variabilidad climática. La variabilidad interanual (estimada por el CV: coeficiente de variación entre años) de la precipitación mensual es mayor en los meses de verano $(\mathrm{CV}=71,74$ y $64 \%$, para diciembre, enero y febrero, respectivamente) que en invierno $(\mathrm{CV}=31,32$ y $24 \%$, para junio, julio y agosto), mientras que la variabilidad interanual de la temperatura media mensual es mayor en otoño e invierno $(\mathrm{CV}=27,25$ y $23 \%$ para

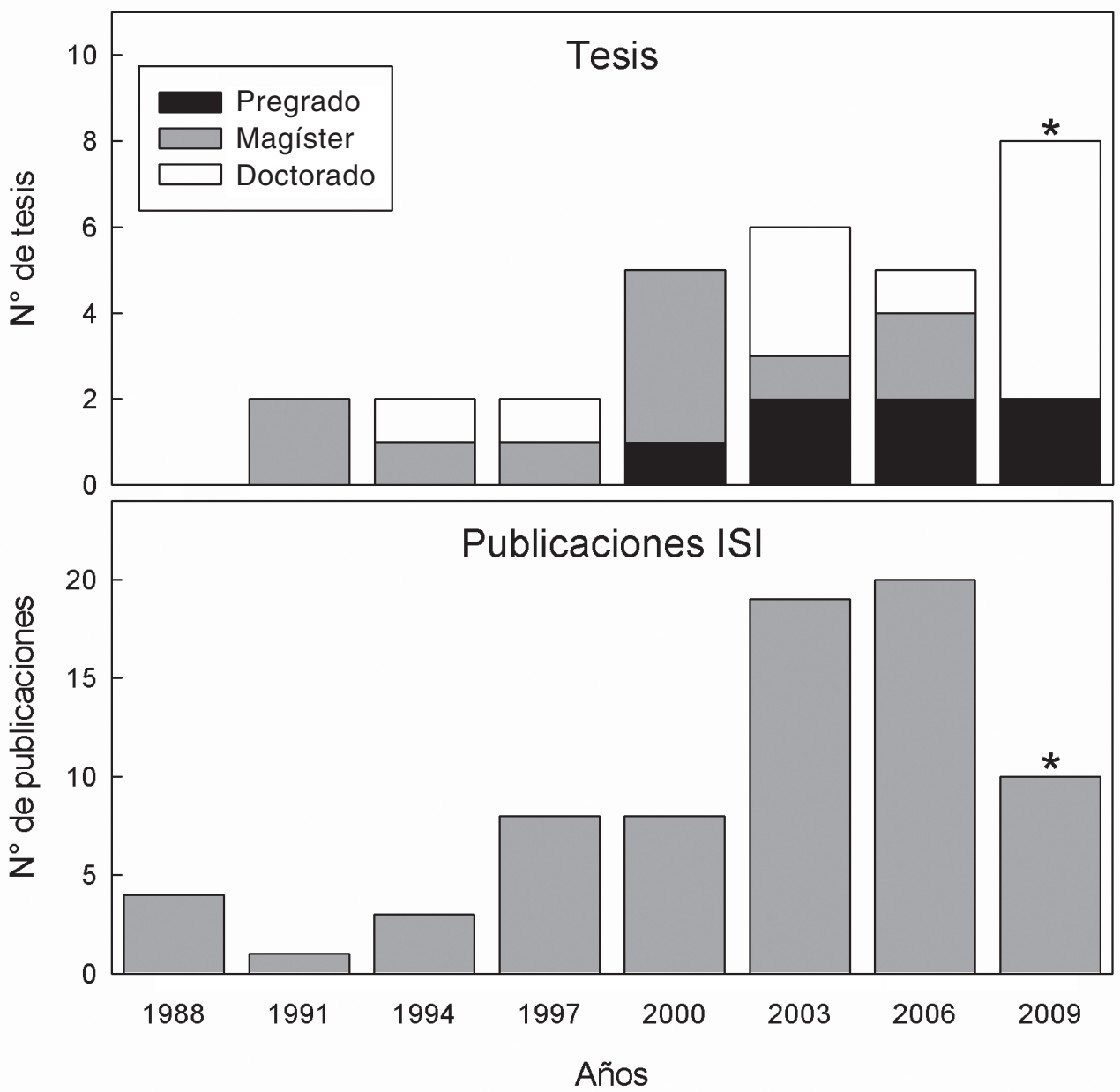

Fig. 2: Número de publicaciones en revistas ISI y tesis (pre y postgrado) generadas a partir de trabajos en la EBSD y sus alrededores, o por investigadores vinculados a ella, en intervalos de tres años, salvo el último $\left(^{*}\right)$ que solo incluye el año 2008 y hasta abril del 2009.

Number of ISI publications and thesis (under and postgraduate) generated from studies within EBSD and its surroudings, or by associate researchers, counted by three years intervals, but the last one $\left(^{*}\right)$ including only year 2008 up to April 2009. 
mayo, junio y julio). Los promedios anuales de temperatura y precipitación hasta la fecha son de $10^{\circ} \mathrm{C}$ y $2,110 \mathrm{~mm}$, respectivamente (Tabla 1). En el largo plazo estos datos pueden descubrir tendencias que no son detectables con datos de corto plazo. En particular, estamos interesados en la variabilidad de la magnitud y frecuencia de precipitaciones de verano, para las cuales se pronostica una declinación en la zona austral de Chile (DGF \& CONAMA 2006), y en comparar estas tendencias con otros registros climáticos de largo plazo de la región (e.g., Puerto Montt). La implementación de la red ESELP permitirá conectar una red de estaciones climáticas a través de una variedad de ecosistemas entre la región semiárida y subantártica, con datos respaldados automáticamente en un servidor central.

\section{Parcelas permanentes y dinámica de bosques}

Las mediciones de largo plazo en bosques naturales son esenciales para detectar tendencias en los ciclos de crecimiento y regeneración, asociadas a fluctuaciones climáticas y/o perturbaciones a escala local, regional o global (Armesto et al. 1996, Garreaud \& Battisti 1999, Gutiérrez et al. 2009). Además, el monitoreo de bosques es

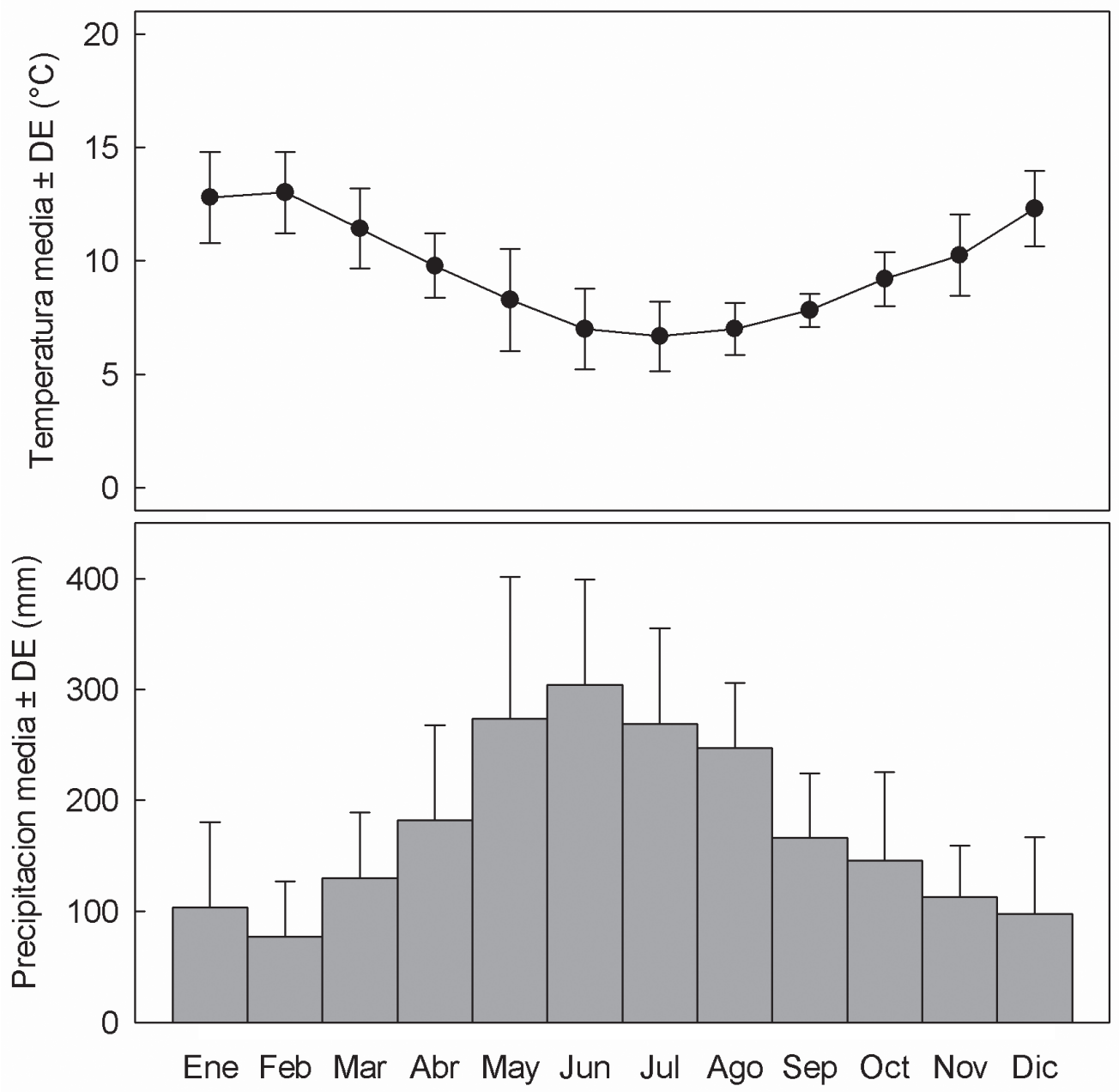

Fig. 3: Promedios mensuales y variabilidad interanual de la temperatura del aire y precipitación según datos de la Estación Meteorológica de la Estación Biológica Senda Darwin (1997-2008). Barras de error: $\pm 1 \mathrm{DE}$ ( $\mathrm{n}=8$-13 años, según disponibilidad de datos).

Monthly averages and inter-annual variability of air temperature and precipitation from data of weather station of EBSD (1997-2008). Error bars: \pm 1 SE ( $=8-13$ years, upon data availability). 
una herramienta fundamental para evaluar la efectividad de las áreas protegidas y medidas de conservación de la biodiversidad, así como las consecuencias del manejo en el largo plazo (Arroyo et al. 1999, Lindenmayer \& Franklin 2002). No obstante, el monitoreo de variables indicadoras del estado de los ecosistemas es una tarea aún no consolidada en el estudio de los bosques nativos chilenos. La red de monitoreo de bosques de la EBSD es una iniciativa pionera para estudiar las respuestas de los bosques nativos a regímenes de perturbación y futuros cambios climáticos.

Las parcelas instaladas en bosques primarios y secundarios de EBSD en 1998 (Aravena et al. 2002), ampliaron una red iniciada en otros bosques de Chiloé en 1989 (Tabla 2). Actualmente, la red de monitoreo en Chiloé cuenta con 10 parcelas en bosques Valdivianos y 13 en Nordpatagónicos (Gutiérrez et al. 2009), cuatro en bosques de alerce y cuatro en ciprés de las Guaitecas. A una escala más amplia, colaborando con otros sitios de estudios de largo plazo, se han instalado parcelas permanentes (PP) en los parques o reservas de Fray Jorge (Gutiérrez et al. 2008a), Los Ruiles y los Queules en Chile central, y en bosques subantárticos del Parque Etnobotánico Omora (Tabla 2). En total, la red liderada por la EBSD incluye un total de $40 \mathrm{PP}$ georeferenciadas, localizadas en áreas protegidas o predios privados (Tabla 2). El protocolo de muestreo se describe en Aravena et al. (2002). En el paisaje rural del norte de la Isla de Chiloé y alrededores de la EBSD, se han identificado bosques antiguos o primarios, con árboles de más de 500 años (Gutiérrez et al. 2004) (Tabla 2) .

Recientemente, el remuestreo de un bosque secundario establecido postincendio en la EBSD (sitio SD1, Aravena et al. 2002) reveló patrones de cambio de la estructura del bosque que refleja las primeras décadas del repoblamiento. La estructura de diámetros (dap) de 1998 muestra una concentración de las especies en las clases diamétricas inferiores (< $10 \mathrm{~cm}$, Fig. 4), y algunos individuos remanentes del bosque original muertos en pie y vivos con dap $>20 \mathrm{~cm}$ (Weinmannia trichosperma Cav. y Nothofagus nitida (Phil.) Krasser). Los remanentes facilitarían el reclutamiento de especies arbóreas a través de un aumento de la lluvia de semillas dispersadas por aves ("efecto percha”). En el área, el establecimiento de plántulas de árboles ocurrió sobre estructuras leñosas remanentes (e.g., tocones y troncos caídos), tal como fue documentado por Papic (2000). Al cabo de una década, los individuos con dap $<10 \mathrm{~cm}$ siguen siendo los más frecuentes, pero aumentó la representación de árboles de clases diamétricas intermedias (10$35 \mathrm{~cm}$, Fig. 4). Drimys winteri J.R. et G. Foster y Nothofagus nitida, especies pioneras de rápido crecimiento en áreas abiertas han predominado en la década de estudio. Además, se registró un incremento de la diversidad de especies de árboles juveniles (dap $<5 \mathrm{~cm}$ ) y de la proporción de especies tolerantes a sombra (e.g., Caldcluvia paniculata (Cav.) D. Don, Saxegothaea conspicua Lindl. y Podocarpus nubigena Lindl.). Registros de largo plazo, complementados por estudios experimentales (Papic 2000, Bustamante, datos no publicados), permitirán entender los patrones $\mathrm{y}$ mecanismos de la dinámica sucesional en bosques en Chiloé.

\section{TABLA 1}

Resumen del registro climático de la EBSD, período 1997-2008 (excluyendo meses con datos faltantes por falla instrumental).

Summary of climate record of EBSD, period 1997-2008 (excluding months with missing data due to instrumental failure).

\begin{tabular}{lc}
\hline Variable & Valor \\
\hline Temperatura media anual $\left({ }^{\circ} \mathrm{C}\right)$ & 9.6 \\
Mínima-Máxima $\left({ }^{\circ} \mathrm{C}\right){ }^{*}$ & $4.0-19.1$ \\
General & $6.1-19.1$ \\
Verano (Dic-Feb) & $4.6-17.0$ \\
Otoño (Mar-May) & $4.0-11.0$ \\
Invierno (Jun-Ago) & $4.0-15.2$ \\
Primavera (Sep-Nov) & 2109.9 \\
Precipitación media anual (mm) & \\
Proporción de precipitación & $13.2 \%$ \\
Verano & $20.2 \%$ \\
Primavera & $27.8 \%$ \\
Otoño &
\end{tabular}

* Considerando el promedio mensual de temperatura mínima y máxima. 
$\underset{\pi}{\pi}: \frac{\pi}{0}$

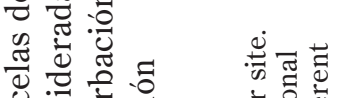

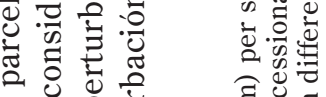

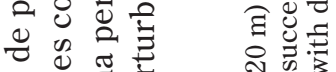

$\circ \circ . \exists$

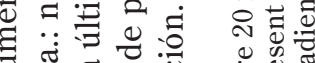

$\stackrel{\Xi}{=} \pi$

II

乙 证讨

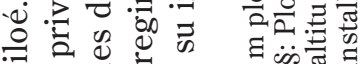

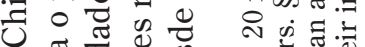

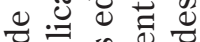

을

氙

응

造

递范范

ए

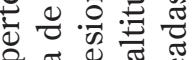

\&

0 o d 0

记

떵 50

$\sim \quad \frac{0}{0}$ 更

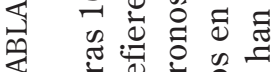

dै

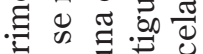

它寻蓄

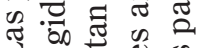

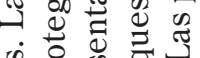

vi

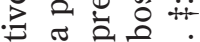

安政毒

क

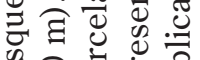

凉完产

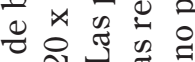

8 证 的

: on

बे एँ

造

导范

语范

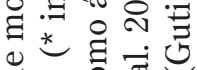

․ हैं त्र

ป⿻

过

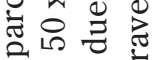

원연

递焉宫

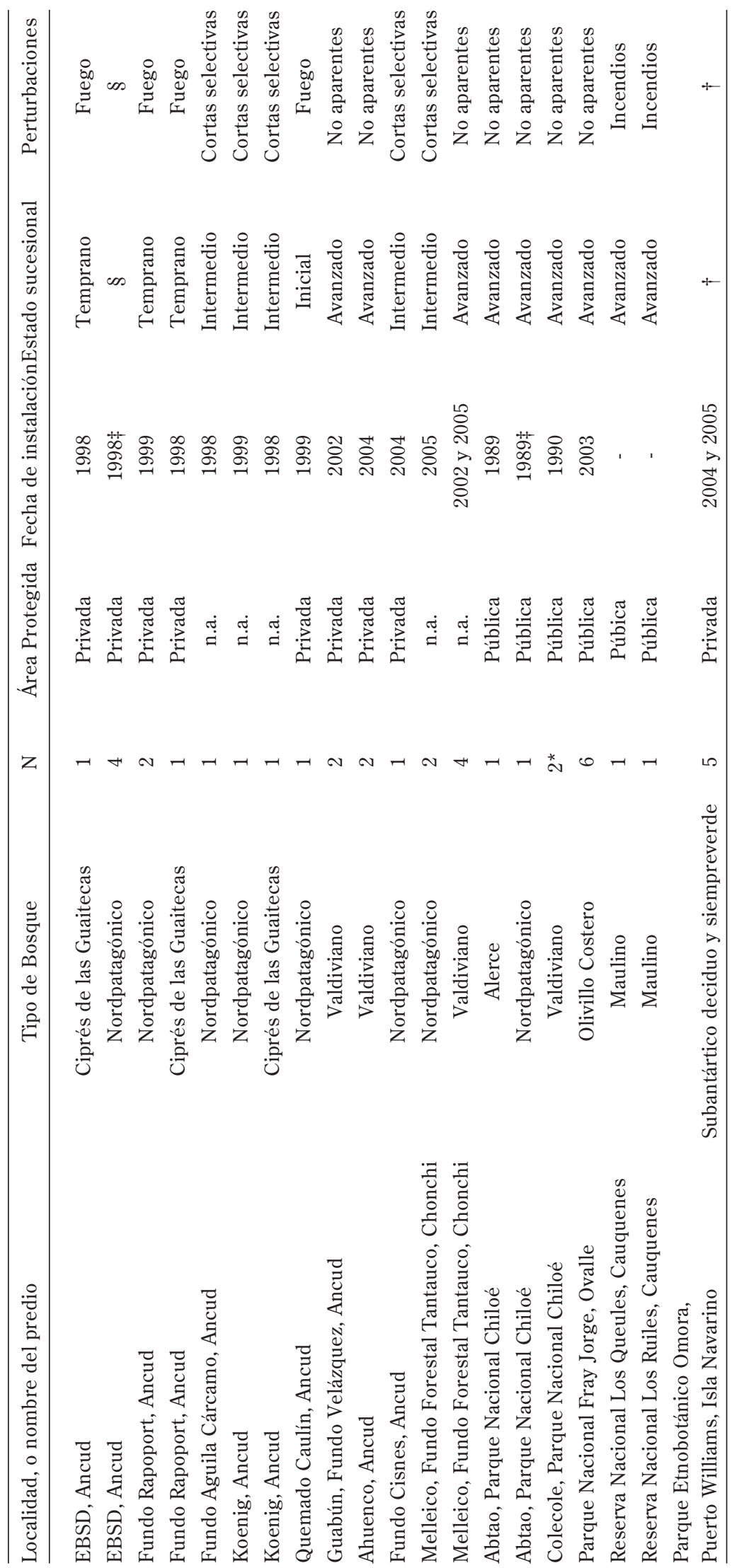


La mayoría de los monitoreos en bosques nativos en Chile corresponden a parcelas en bosques secundarios que han sido manejados o degradados por extracción de leña, madera y/o pastoreo (Lara et al. 2000). Aunque el muestreo periódico de bosques secundarios es muy relevante para evaluar prácticas silvícolas y sus efectos, es necesario establecer parcelas en bosques antiguos, en ausencia de grandes impactos humanos, para evaluar predicciones sobre los mecanismos físicos (e.g., perturbaciones y tendencias climáticas) y demográficos (e.g., mortalidad y reclutamiento) que afectan la estructura y composición de los bosques (Gutiérrez et al. 2009).

El monitoreo de PP en bosques permite poner a prueba hipótesis acerca de la estabilidad, resiliencia de los bosques frente a diferentes perturbaciones (Hendrickson 2003). Por ejemplo, es posible documentar efectos colaterales de la fragmentación sobre la persistencia de interacciones planta-animal y estructura de los bosques (Laurance et al. 1998, Gutiérrez et al. 2009). También se puede evaluar la importancia de elementos estructurales de bosques antiguos, como árboles viejos y troncos caídos, para la conservación de la biodiversidad, dinámica y funciones ecosistémicas (Díaz et al. 2005, 2006, Jaña-Prado 2006, 2007, Carmona et al. 2002, Christie \& Armesto 2003).
Un subconjunto de la red de PP (Tabla 2) ha hecho posible conocer los cambios de variables edáficas (e.g., relación carbono:nitrógeno) y procesos biogeoquímicos (e.g., fijación no simbiótica de nitrógeno, mineralización neta de nitrógeno) a lo largo de una cronosecuencia sucesional (Aravena et al. 2002, Pérez et al. 2004, Carmona 2004). Estos estudios revelaron una gran resiliencia de estos procesos a la perturbación por incendios moderados de origen antropogénico. Comparaciones de procesos biogeoquímicos del suelo en bosques intactos y sometidos a manejo forestal indican disrupciones de algunas funciones básicas (Pérez et al. 2009). Combinando estos estudios con el monitoreo demográfico permiten comprender procesos de la interfase planta-suelo y sus efectos sobre la dinámica del rodal.

\section{Ecología y manejo de turberas}

Las turberas dominadas por musgos del género Sphagnum son humedales caracterizados por acumular profundos depósitos de materia orgánica en descomposición, con escaso o nulo crecimiento de plantas leñosas. Se distribuyen en regiones con altas precipitaciones $(>2000$ $\mathrm{mm}$ ), en depresiones del terreno con drenaje pobre, donde se acumula agua o esta fluye lentamente. Las turberas desempeñan

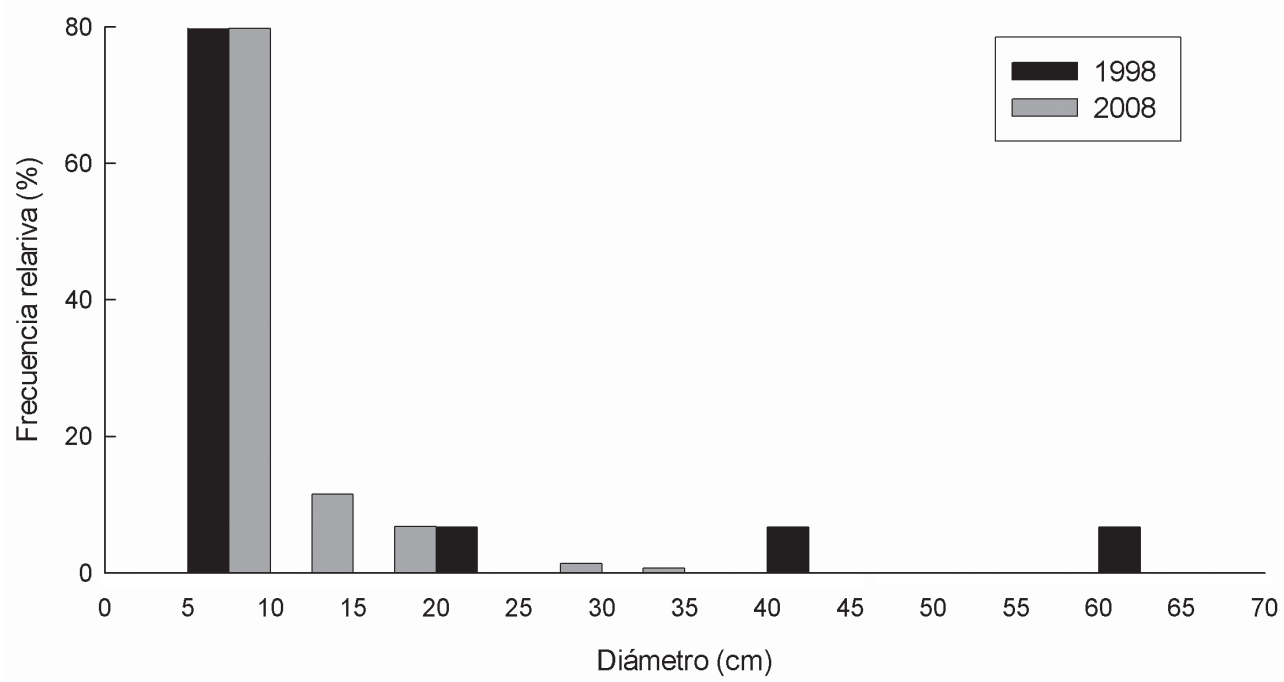

Fig. 4: Cambios en la estructura de diámetros de un bosque secundario de la EBSD después de 10 años de sucesión postincendio.

Changes of diameter structure of a secondary forest of EBSD after 10 years of post-fire succession. 
funciones ecológicas claves, ya que almacenan agua de lluvia y deshielo, mantienen la calidad del agua que drena los esteros, y almacenan grandes cantidades de carbono orgánico de lenta descomposición (Clymo \& Hayward 1982, Clymo et al. 1998, Gorham 1991, Moore et al. 1998).

En Chiloé existen dos tipos de turberas: naturales o primarias y secundarias, generadas por acción del ser humano, también llamadas turberas antropogénicas y conocidas localmente como "pomponales" (Díaz et al. 2008). Las turberas naturales tienen acumulaciones de turba superiores a $50 \mathrm{~cm}$ de profundidad y datan desde el último ciclo glacial (18 a 20 mil años AP), cuando las turberas ocuparon gran parte del territorio de Chiloé (Villagrán 2001). Las turberas antropogénicas presentan menor acumulación de turba y se forman sobre el suelo mineral después de incendios de bosques o grandes talas rasas que datan del siglo XX. Este tipo de turberas secundarias están presentes en la EBSD (Fig. 1).

Por casi dos décadas, agricultores de la Región de Los Lagos han utilizado las turberas para extraer musgo (Sphagnum magellanicum Brid.) que se exporta deshidratado y prensado como material para viveros y otros usos. La extracción de musgo actualmente no está regulada, debido a que ninguna agencia estatal o privada reconoce responsabilidad sobre el recurso, generando un proceso de degradación paulatina de estos ecosistemas.

Desde el año 2004, hemos evaluado el estado de este ecosistema con el fin de proponer mejores formas de uso. Hemos producido dos documentos disponibles en nuestro sitio web (http://www.sendadarwin.cl), uno sobre la importancia de las turberas y el pompoñ (Sphagnum) en la Isla de Chiloé y una guía de campo de la exclusiva y rica flora de cerca de 30 especies que crece sobre las turberas. Se han realizado talleres de capacitación en el conocimiento de la flora e hidrología de las turberas con dos comunidades Huilliches (Púlpito y Romazal), además de talleres y cursos de capacitación para organismos públicos, un simposio y un seminario internacional en la ciudad de Puerto Montt para discutir aspectos legales y ambientales de la sustentabilidad de las turberas (Díaz et al. 2008).
Sobre estas bases, se generó e implementó un modelo productivo y comercial en los humedales de la región. Junto con la consultora Surambiente Ltda., Cooperativa San Andrés y Asociación Gremial de Productores de Musgo ponpon. Se realizaron experimentos de campo en parcelas de los mismos agricultores para estimar el crecimiento y productividad postcosecha del musgo (Díaz \& Tapia, datos no publicados). Además, se proporcionó asesoría técnica a agricultores para avanzar hacia un manejo sustentable de sus humedales mediante protocolos regulados de extracción y asesoría en la exportación (Díaz 2008).

Para continuar esta investigación, donde se obtuvieron los primeros datos sobre crecimiento y productividad en áreas manejadas y no manejadas, desde noviembre de 2008 se está evaluando la regeneración, crecimiento y productividad de Sphagnum bajo distintas condiciones de luz y humedad. Estos experimentos, en los que participan agentes públicos, privados y la comunidad, generan nuevo conocimiento científico con relevancia social, sentando las bases para un manejo razonable de este recurso natural ausente de la atención de las instituciones ambientales del Estado.

\section{Ecología de matorrales sucesionales}

En las últimas décadas, bosques nativos de Chiloé han sido masivamente reemplazados por matorrales sucesionales, humedales, con escasa regeneración arbórea, y praderas antropogénicas (Díaz 2004, Echeverría et al. 2007). El estudio de la dinámica de regeneración de áreas degradadas producto de actividades antrópicas ha sido un foco de las investigaciones en la EBSD. Aunque muchos matorrales están rodeados a corta distancia $(<300 \mathrm{~m})$ por bosques que constituyen fuentes de propágulos, hemos constatado que han permanecido sin colonización arbórea por al menos 50-60 años. Los principales colonizadores arbóreos, Drimys winteri (Winteraceae) y Nothofagus nitida (Nothofagaceae) están a menudo ausentes de estas áreas. Nuestro interés ha sido conocer los factores bióticos y abióticos que limitan la colonización.

Alteraciones del ciclo hidrológico asociadas con el cambio del uso del suelo desde bosque a matorral o pradera (Díaz et al. 2007) 
sugieren que un filtro ambiental (sensu Fattorini \& Halle 2004) que limita la colonización se debe al anegamiento estacional de los suelos. Los niveles de la napa freática son entre 10 y $50 \mathrm{~cm}$ más altos en los suelos del matorral que en bosques aledaños, incluso durante los meses de verano (Díaz et al. 2007). El establecimiento de plántulas arbóreas ocurre exclusivamente sobre montículos elevados formados por detritus leñoso, sugiriendo que es necesaria la elevación de las plantas por el nivel freático (Papic 2000).

Las semillas de muchas especies arbóreas de Chiloé son activamente dispersadas por aves frugívoras y no presentan latencia (Armesto \& Rozzi 1989, Willson et al. 1996). La lluvia de semillas es un factor limitante en el proceso de colonización arbórea de praderas o matorrales antropogénicos (Jaña-Prado 2007), independiente de la distancia al bosque. La vegetación no arbórea que sobrevive la perturbación o se establece en los primeros años puede facilitar este proceso. Matorrales secundarios dominados por el arbusto Baccharis patagonica $\mathrm{H}$. et $\mathrm{A}$. reciben menos lluvia de semillas de árboles que matorrales dominados por dos especies arbustivas de Berberis (Bustamante-Sánchez \& Armesto, datos no publicados). Esta diferencia puede estar determinada por la actividad y conducta de las aves frugívoras, que visitan preferentemente arbustos con frutos carnosos (Berberis spp.), en contraste a arbustos con semillas secas. (Armesto et al. 2001, JañaPrado 2007).
En la fase postdispersión, la depredación de semillas por aves y roedores y la facilitación son importantes moduladores del reclutamiento arbóreo en áreas de matorral y praderas. Matorrales secundarios dominados por la especie pionera $B$. patagonica presentan mayor depredación de semillas que praderas (Tabla 3), limitando el establecimiento de plántulas arbóreas. La especie pionera Embothrium coccineum J.R. et G. Foster germinó y fue capaz de establecerse en áreas abiertas cubiertas por el musgo Sphagnum magellanicum y bajo arbustos (Díaz \& Armesto 2007), mientras la germinación y/o sobrevivencia de Amomyrtus luma Mol. y Eucryphia cordifolia Cav. solo tuvo lugar bajo un dosel arbustivo (Bustamante-Sánchez et al., datos no publicados).

Hasta la fecha hemos identificado algunos de los procesos y mecanismos que retardan o facilitan la regeneración en áreas sucesionales, a través de estudios de una o dos temporadas (e.g., Díaz \& Armesto 2007, Armesto et al. 2001, Jaña-Prado 2007, Bustamante-Sánchez et al., datos no publicados). Es necesario extender estos estudios a escalas temporales más congruentes con la dinámica sucesional, que permitan capturar efectos de la variabilidad temporal en clima y fecundidad de los árboles sobre la regeneración (Clark et al. 1999). La naciente red Chilena de ESELP posibilitará estudios a escala decadal de las dinámicas poblacionales de especies pioneras y tardías a lo largo de la sucesión.

TABLA 3

Porcentaje medio de remoción o depredación de semillas de ocho especies leñosas principalmente por roedores y aves granívoras en un matorral de Baccharis patagonica y en una pradera. Se instalaron estaciones (una placa petri por cada especie, con 10 semillas cada una) durante el verano y otoño de 2005. El número de semillas remanentes se contó después de cinco noches de exposición a los granívoros. En paréntesis se muestra el error estándar.

Mean percentage of seed predation, principally by rodents and granivorous birds, of eight woody species in a shrubland of Baccharis patagonica and a pastures. Seed predation stations were installed (one petri dish per species with 10 seeds each one) during the summer and fall of 2005 . The number of seeds left was counted after five nights of exposure to the granivores. Between parentheses is shown the standard error.

\begin{tabular}{lcc}
\hline & Verano & Otoño \\
\hline Matorral & & $87.3(2.7) \mathrm{N}=105$ \\
Pradera & $29.2(4.1) \mathrm{N}=92$ & $66.8(4.1) \mathrm{N}=105$ \\
Prueba de Kruskal-Wallis & $5.3(1.9) \mathrm{N}=93$ & $\mathrm{H}_{(1,210)}=11.14 ; \mathrm{P}<0.001$ \\
\hline
\end{tabular}




\section{Estudio experimental del cambio climático}

Tanto los modelos de cambio climático para la zona templada del sur de Sudamérica como los datos instrumentales, predicen disminuciones e irregularidad de las lluvias estivales para las próximas décadas (DGF \& CONAMA, 2006). Por esto, en el año 2007 iniciamos un experimento de largo plazo que simula una reducción de magnitud 20-30 \% de la precipitación de verano en dos bosques secundarios de 60-80 años (dosel de 15-20 m) dominados por Nothofagus nitida, Drimys winteri, y Caldcluvia paniculata. Para este fin se instaló un sistema experimental que excluye un porcentaje de la lluvia que escurre a través del dosel en el interior de parcelas de 20 x 20 $\mathrm{m}$, comparadas a dos parcelas control de las mismas dimensiones. Este estudio de largo plazo sigue un protocolo similar a un experimento de exclusión total de lluvia en una hectárea de bosque tropical estacional de Brasil (Nepstad et al. 2002). Hasta este momento se ha aplicado el tratamiento de exclusión de lluvia durante dos veranos consecutivos en uno de los bosques, y se han evaluado las respuestas de la vegetación (e.g. tasa de crecimiento radial de los árboles, cobertura de helechos, caída de hojarasca) y el suelo (e.g., tasas de respiración del suelo, descomposición de hojarasca). Se mantienen registros de la humedad del suelo y temperatura del aire durante todo el año, incluyendo el periodo estival en parcelas control y con exclusión. Este experimento busca determinar la resiliencia de los procesos ecosistémicos y poblacionales del bosque templado al cambio climático, especialmente considerando que la sequía estival es un factor limitante del reclutamiento arbóreo (Papic 2000)

\section{Chucaos: Biología poblacional y conservación}

El chucao (Scelorchilus rubecula [Kittlitz]; Rhinocryptidae) (Fig. 5A) es un ave emblemática y endémica de los bosques lluviosos del sur de Chile y suroeste de Argentina en hábitats con densa cobertura vegetal (Willson 2006). Anidan en cavidades, ocupando agujeros en árboles vivos y muertos, troncos caídos, montículos de tierra y ocasionalmente acumulaciones de hojarasca y cúmulos de epífitas (DeSanto et al. 2002). Debido a que es una especie sensible a la pérdida y fragmentación del bosque (Sieving et al. 2000) pueden servir como referente para la conservación de otras especies restringidas al bosque. Durante 15 años se estudiaron las poblaciones de chucao en la EBSD y fragmentos de bosque del sector rural, documentando aspectos desconocidos de su biología e historia natural, y los efectos de la fragmentación y pérdida de cobertura del bosque sobre su éxito reproductivo y su movilidad a través del paisaje (e.g., Willson et al. 1994, Willson 2006).

Los chucaos son aves no migratorias que habitan el sotobosque (primeros $2 \mathrm{~m}$ verticales del bosque) y que rara vez vuelan largas distancias. Se alimentan de invertebrados de la hojarasca del bosque, y algunas presas mayores como pequeños anfibios o frutos sobre el suelo (Willson 2006). Son muy territoriales y cada pareja defiende un territorio promedio de 1 ha. Ocasionalmente, conflictos territoriales derivan en un combate físico, especialmente en la temporada de nidificación. Los chucaos alcanzan su adultez al año después de nacer, son socialmente monógamos y ambos padres cuidan a sus crías. Un $44 \%$ de los adultos sobreviven desde la edad de un año a la edad de dos años, pero desde los dos a los siete años la sobrevivencia alcanza un $72 \%$ (Willson \& Pendleton 2008). La longevidad promedio es 2-3 años, aunque pueden vivir hasta siete.

Los depredadores del chucao incluyen aves rapaces diurnas y nocturnas. El chucao a menudo despliega señales de alarma si visualiza un chuncho (Glaucidium nanum [King]) y esconde a sus polluelos si detecta a un peuquito (Accipiter chilensis [Vieillot]) o un concón (Strix rufipes King) en la vecindad de su nido. Perros y gatos domésticos constituyen una amenaza seria para esta especie en el paisaje rural. Los principales depredadores de nidos son probablemente roedores y el monito del monte (Dromiciops gliroides) (Willson 2006). El éxito reproductivo es mayor en nidos protegidos por largos túneles de entrada. El tamaño de la nidada es dos y ocasionalmente tres huevos en nidos en bosques fragmentados del paisaje rural del norte de Chiloé. El éxito de los nidos de chucaos fue c. $72 \%$ en bosque continuo y $63 \%$ en bosque fragmentado, 
comparado con $20-30 \%$ en aves de nidos abiertos en la misma zona (DeSanto et al. 2002, Willson et al. 2005). La tala de bosques sigue siendo una amenaza para los chucaos, debido a que la extracción de los grandes árboles implica la pérdida de sitios seguros de nidificación. La tala, el pastoreo y pisoteo del ganado destruyen la cobertura del sotobosque, aumentando la exposición a depredadores y reduciendo la producción de hojarasca, donde los chucaos encuentran su alimento.

Algunos fragmentos de bosque del sector rural están completamente rodeados por praderas, que los aíslan de otros parches de bosque. Debido a que las praderas constituyen barreras para la dispersión de los chucaos, el aislamiento aumenta su potencial de consanguinidad y disminuye su éxito reproductivo (Castellón \& Sieving 2006a, 2006b, Willson 2004). Juveniles marcados tienen menor probabilidad de dispersarse cuando nacen en fragmentos rodeados por praderas que cuando nacen en fragmentos de bosque conectados por corredores densos de vegetación. Un $21 \%$ de juveniles nacidos en fragmentos aislados permanecieron allí hasta la madurez, comparado con solo un $3 \%$ en fragmentos conectados. Además, un $18 \%$ de los machos en fragmentos aislados no lograron conseguir pareja, en tanto que en fragmentos conectados solo $1 \%$ no se reprodujo. Los corredores de vegetación densa usados por los chucaos (Sieving et al. 2000) pueden tener unos pocos metros de ancho $(3-5 \mathrm{~m})$, cuando las
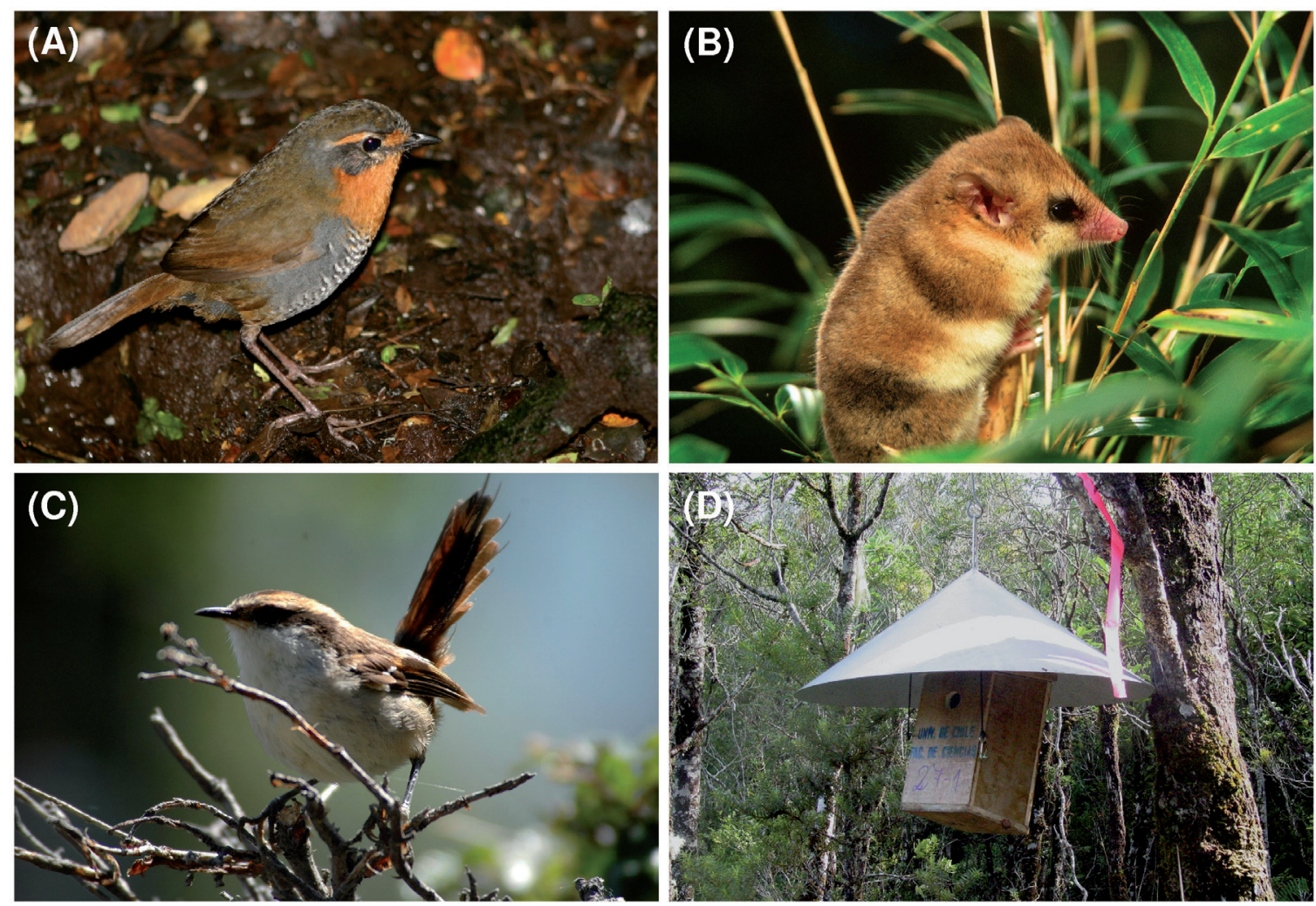

Fig. 5: Especies de aves y mamíferos más intensamente estudiadas en la EBSD y la red ESELP. A: Chucao (Scelorchilus rubecula; fotografía: Juan Luis Celis). B: Monito del monte (Dromiciops gliroides; fotografía: Andrés Charrier). C: Rayadito (Aphrastura spinicauda; fotografía: Juan José Sanz). D: Caja anidera para estudios en rayadito y otras aves de los bosques templados, con protección metálica para evitar el ingreso del monito del monte, un depredador de aves y polluelos.

Bird and mammal species most intensively studied in EBSD and the LTSER network: A: Chucao (Scelorchilus rubecula). Photography: Juan Luis Celis. B: Monito del monte (Dromiciops gliroides). Photography: Andrés Charrier. C: Rayadito (Aphrastura spinicauda). Photography: Juan José Sanz. D: Nesting box for rayadito studies and other temperate forest birds, with protection against monito del monte, an egg and chick predator. 
distancias de dispersión son del orden de 10100 metros. Sin embargo, en sectores donde las áreas abiertas son extensas y los fragmentos de bosque son escasos la dispersión es menos probable y el riesgo para las poblaciones de Chucaos (y otras aves de sotobosque) es mayor (Castellón \& Sieving 2007).

Estudios de largo plazo de esta especie endémica han permitido proponer diseños de paisaje que facilitan la persistencia de poblaciones viables en ambientes rurales (Willson \& Armesto 2003). La implementación de estos diseños requiere la participación de actores sociales y políticos, principalmente los propietarios de tierras rurales.

Demografía y conservación del monito del monte (Dromiciops gliroides)

El monito del monte (Dromiciops gliroides) (Fig. 5B), marsupial endémico de los bosques templados lluviosos de Sudamérica austral (Hershkowitz, 1999), es una de las especies menos conocidas entre los mamíferos de Chile (Celis-Diez et al. 2010). Su singularidad radica en que es considerado un "fósil viviente" por ser el único representante vivo del Orden Microbiotheria, una línea filogenéticamente primitiva de los marsupiales (Palma \& Spotorno 1999). Su distribución está restringida a los bosques templados de Chile y Argentina (Lobos et al. 2005), desde el bosque Maulino ( $\left.35^{\circ} \mathrm{S}\right)$ hasta Chiloé continental, incluyendo la isla de Chiloé $\left(43^{\circ} \mathrm{S}\right)$. Actualmente, D. gliroides es una especie potencialmente amenazada de acuerdo a UICN debido a la pérdida de su hábitat. Estudios de esta especie realizados en la EBSD abarcan relaciones filogeográficas entre poblaciones de Chiloé y del continente, aspectos básicos de su biología, conducta y cuantificación de su densidad poblacional en fragmentos de bosque primario y sometidos a extracción selectiva. Estos estudios han documentado que tanto las poblaciones de la isla de Chiloé como de la Región de Los Lagos y de Bariloche (Argentina) en el continente son derivadas de poblaciones ancestrales ubicadas más al norte (Himes et al. 2008) en la costa de Chile, producto de la historia glacial de los bosques (Villagrán 2001).

Dromiciops gliroides es el único mamífero sudamericano que hiberna (Bozinovic et al.
2004), cuando en invierno escasea su alimento constituido por insectos y frutos. En este período nidifica en cavidades naturales en troncos o nidos construidos con ramas, hojas de quila y especies de musgos y helechos en el denso sotobosque (Jiménez \& Rageot 1979, Celis-Diez et al. 2010).

Desde noviembre de 2005 se mantiene un programa de monitoreo anual de poblaciones de Dromiciops gliroides en parches de bosques y matorrales del paisaje rural cercano a la EBSD, con el fin de estimar tendencias demográficas en el largo plazo. Hasta la fecha se han estimado densidades comparables en parches de bosques primarios y en bosques sujetos a tala selectiva, pero solo presencia marginal en matorrales (Celis-Diez et al. 2010). En ambos tipos de bosque las tasas de crecimiento poblacional son positivas, y las poblaciones tienden a aumentar en periodos estivales en comparación con el periodo invernal previo.

No se detectaron diferencias entre bosques primarios o sujetos a tala selectiva en la probabilidad de sobrevivencia de Dromiciops durante el período 2005-2009 a pesar de que la densidad de árboles grandes $(>30 \mathrm{~cm}$ de diámetro del tronco), como un indicador de abundancia de cavidades naturales disminuye a la mitad en bosques expuestos a tala selectiva. La alta densidad de quila en bosques perturbados, como consecuencia de la apertura del dosel, mitigaría en parte la ausencia de sitios de anidación, al mismo tiempo que proveería protección contra los depredadores.

A nivel parasitológico, hemos documentado tres especies de ectoparásitos de $D$. gliroides. Dos especies de pulga (Chiliopsylla allophyla [Rothschild] y Plocopsylla diana Beaucournu et al.) presentes también en roedores, y el primer registro en Chile de la garrapata Ixodes neuquenensis Ringuelet, específica de $D$. gliroides (Marín-Vial et al. 2007). También se registró un parásito digestivo, el nemátodo Pterygodermatites sp. (Rictulariidae) y la presencia de un nuevo hemoparásito, una especie ancestral dentro del grupo Hepatozoon sp., que habría evolucionado en conjunto con Dromiciops (Merino et al. 2009). Su potencial vector específico, en base a evidencia genética, sería la garrapata Ixodes neuquenensis (Merino et al. 2009). 
El zorro de Darwin, un carnivoro emblemático de Chiloé, hoy en peligro crítico

Entre las tres especies de zorros chilenos, el zorro de Darwin (Lycalopex fulvipes Martin) es la más pequeña y la única endémica de Chile. Este zorro, debido a sus bajos números poblacionales, a su distribución casi exclusivamente insular y la pérdida de su hábitat es considerado en peligro crítico de extinción (Jiménez et al. 2004). Entre los mamíferos chilenos, y entre los cánidos a nivel mundial, es una de las especies con mayor prioridad de conservación (Cofré \& Marquet 1998, Macdonald et al. 2004). Hasta hace pocos años fue considerado una subespecie de la chilla (Lycalopex griseus [Gray]) presente solo en la Isla Grande de Chiloé (Osgood 1943, Miller et al. 1983), hasta que se encontró una población pequeña en la Cordillera de Nahuelbuta, unos $600 \mathrm{~km}$ al norte de Chiloé (Medel et al. 1990). Actualmente, es considerado una especie genéticamente diferente y ancestral de los cánidos sudamericanos, que tuvo una distribución más amplia en el pasado (Yahnke et al. 1996). A su vez, es el carnívoro terrestre más grande de la isla, con un importante papel en la trama ecológica de los bosques y la cultura rural de Chiloé.

Desde mediados de la década de los 70, hemos estudiado su distribución, hábitos alimenticios, preferencias de hábitat, uso del espacio, estructura social y su relación con el impacto humano en el paisaje de Chiloé. A pesar que fue descrito sobre la base de un ejemplar colectado por Charles Darwin en 1834, el conocimiento de su ecología e historia natural fue hasta hace poco muy pobre (Osgood 1943, Medel et al. 1990). Actualmente, se le detecta en paisajes rurales de Chiloé que contengan fragmentos de bosque nativo, desde el nivel del mar hasta las cumbres de la cordillera costera o de Piuché (650 m) y desde los alrededores de EBSD en la zona norte hasta los bosques de Iñío en el extremo sur de la isla (Jiménez et al. 2005¹).

1 JIMÉNEZ JE, P RUTHERFORD, C BRICEÑO, E LEEGWATER \& SM FUNK (2005) The critically endangered Darwin's fox (Pseudalopex fulvipes), where and how many are left? Combining field data and GIS modelling, XIX Congreso anual de la Society for Conservation Biology, Brasilia, Brazil.
Aunque se pensaba que la especie era dependiente de bosques nativos maduros (Miller et al. 1983), estudios con radiotelemetría en al menos seis localidades de toda la isla y en 35 individuos diferentes demuestran que hoy el zorro habita el mosaico de bosques maduros y secundarios, mezclados con matorrales y áreas abiertas que dominan el paisaje de Chiloé; se le ve también en dunas costeras y playas, donde encuentra alimento, independiente del grado de perturbación del hábitat (Jiménez et al., datos no publicados). $\mathrm{Su}$ alimentación es variada y oportunista, incluyendo pudúes, micromamíferos, aves, insectos, invertebrados costeros, algas, mariscos y frutos carnosos dependiendo del lugar y la estación del año (Jiménez \& Rau $1999^{2}$, Jiménez 2007). Durante el otoño consume gran cantidad de frutos y dispersa semillas de peta (Myrceugenia planipes [H. et A.]), chupón de las rocas (Fascicularia bicolor (R. et P.) Mez) y chupón común (Greigia sphacelata (R. et P.) Regel), a distancias $>700$ $m$ desde las fuentes (Jiménez 2007). También dispersa frecuentemente semillas de Uncinia sp. pegadas al pelo.

El zorro de Darwin está activo todo el día, presenta bajo dimorfismo sexual y su color y morfología corporal son propias de mamíferos de bosques húmedos (Jiménez \& McMahon 2004, Jiménez 2007). A diferencia de la mayoría de los cánidos, no defienden territorios exclusivos y sus ámbitos de hogar presentan alta sobreposición entre machos y hembras de diferentes edades (Killian 2005, Jiménez 2007). El impacto humano sobre los bosques nativos no afecta su presencia cuando el grado de fragmentación de bosques es bajo. Actualmente, el mayor peligro para la conservación de esta especie es la persecución directa por el hombre y sus mascotas (Jiménez \& McMahon 2004). Los campesinos chilotes tienen escaso conocimiento de su biología y sus percepciones son negativas hacia el zorro (Díaz 2005). Es frecuente que zorros de Darwin sean cazados por humanos o muertos por sus perros. Además, hemos encontrado ecto (González-Acuña et al. 2007) y

JIMÉNEZ JE \& JR RAU (1999) Dieta del zorro de Darwin en Chiloé: Variabilidad espacial y temporal. Resumen, II Taller de Carnívoros, organizado por el Servicio Agrícola y Ganadero, Coihaique. 
endoparásitos (Jiménez et al., datos no publicados) compartidos con los perros domésticos, quienes pueden transmitir enfermedades virales, como distemper canino (Jiménez et al., datos no publicados). La conservación y viabilidad de esta especie amenazada en Chiloé dependerá fuertemente del manejo del paisaje rural y de la tenencia responsable de perros en las comunidades rurales.

Estudio de largo plazo del rayadito (Aphrastura spinicauda)

Desde el año 2002 hemos realizado estudios de largo plazo sobre una de las aves más frecuentes del bosque nativo (Rozzi et al. 1996, Cornelius et al. 2000), el rayadito o yiqui-yiqui (Aphrastura spinicauda; Furnariidae) (Fig. 5C), en la EBSD. Dichas investigaciones se extendieron en años posteriores a otros sitios de la naciente red ESELP (Parque Etnobotánico Omora y Parque Nacional Fray Jorge), con el fin de conocer la variabilidad intraespecífica a nivel fenotípico, genético e histórico en un contexto geográfico. Esta ave es endémica de los bosques templados australes de Chile y Argentina y habita desde el Parque Nacional Fray Jorge $\left(30^{\circ} \mathrm{S}\right)$ hasta el Cabo de Hornos (56 ${ }^{\circ}$ S) (Johnson \& Goodall 1967, Remsen 2003, Rozzi 2003). Es un ave pequeña (11-13 g), principalmente insectívora, ocupante secundaria de cavidades en árboles para anidar; vive en grupos conspicuos durante la época no reproductiva (Vuilleumier 1967, Grigera 1982, Ippi \& Trejo 2003, Moreno et al. 2005, Cornelius 2008). Dado su extenso rango latitudinal (c. $2,800 \mathrm{~km}$ ), las poblaciones experimentan condiciones ambientales y presiones selectivas contrastantes. En este sentido, su estudio puede contribuir a comprender las causas de cambios microevolutivos e incluso procesos de especiación o subespeciación (e.g., Foster 1999, Quispe et al. 2009). Con el objeto de estudiar algunos rasgos de historia de vida del rayadito, se instalaron cajas anideras (Fig. 5D) en bosques de la EBSD y el fundo Los Cisnes en la zona norte de Chiloé, las cuales son monitoreadas anualmente durante la temporada reproductiva. Durante el período reproductivo adultos y polluelos utilizan dichas cajas nido, por lo que es posible marcarlos para su seguimiento e identificación posterior. Así podemos registrar el tamaño y períodos de puesta (Moreno et al. 2005), el dimorfismo sexual en el cuidado parental, diferencias morfológicas (Moreno et al. 2007), datos parasitológicos (Merino et al. 2008) y rasgos conductuales (Moreno et al. 2007, Ippi et al. y Vásquez et al., datos no publicados).

Hasta la fecha (2008), en bosques del sector rural de la Isla de Chiloé, el rayadito puede ser caracterizado como una especie con alta inversión parental, cuatro (valor modal) huevos grandes en relación a su tamaño corporal y un largo período de dependencia de los polluelos, caracteres más similares a los de especies de aves tropicales que a los de aves que habitan bosques en latitudes altas del hemisferio norte (Moreno et al. 2005). Ambos padres presentan escaso dimorfismo sexual y participan por igual de la incubación y alimentación de los polluelos (Moreno et al. 2007), así como de la defensa del nido (Ippi et al., datos no publicados).

Dado que el rayadito pertenece a una de las familias de aves más diversas del planeta (más de 200 especies) y al suborden Suboscine, uno de los menos conocidos (Irestedt et al. 2001, Jaramillo 2003, Remsen 2003), la información de largo plazo sobre su ecología e historia evolutiva podría contribuir a descubrir nuevas formas mediante las cuales los organismos enfrentan condiciones ambientales diversas 0 cambiantes en el tiempo y espacio. Este trabajo ofrece una novedosa base teórica aplicable al estudio de muchas especies que habitan en Sudamérica. Por otro lado, el estudio de largo plazo de especies dependientes de bosques, como el rayadito, permitiría, establecer modelos o indicadores para políticas de conservación y/o manejo de otras especies filogenéticamente cercanas (véase Vásquez \& Simonetti 1999), pero menos conocidas, que utilizan cavidades naturales en grandes árboles de los bosques nativos.

Reconociendo los vínculos entre biodiversidad y estructura de los bosques

Muchos bosques templados y tropicales se caracterizan por la presencia de estructuras físicas y biológicas de grandes dimensiones como son árboles emergentes del dosel, muertos en pie, troncos caídos, tocones, y parches de vegetación densa en el sotobosque 
(e.g., Aravena et al. 2002, Carmona et al. 2002, Gutiérrez et al. 2009, Van Pelt 2007). Estas estructuras proveen hábitats y recursos para diversas especies de plantas y animales en los ecosistemas (Franklin et al. 1981). Los bosques templados antiguos del sur de Chile presentan típicamente un dosel multiestratificado (Gutierrez et al. 2009), dominado por especies arbóreas siempreverdes de hojas anchas, con troncos cargados de epífitas y enredaderas, frecuentemente con un denso sotobosque de quila (bambú) en claros del dosel formados por caída de árboles (Gutiérrez et al. 2008b). Reid et al. (2004) mostraron que las aves del sotobosque habitan bajo la cobertura de quila (Chusquea sp.), donde se refugian de los depredadores y obtienen alimento. Dos especies de aves del sotobosque, el colilarga (Sylviorthorhynchus desmursii Des Murs, Furnariidae) y el churrín de la Mocha (Eugralla paradoxa (Kittlitz), Rhinocryptidae) pasan la mayor parte de su ciclo de vida en los densos parches de quila (McPherson 1999, Díaz et al. 2006). Otras tres especies, el chucao (Scelorchilus rubecula), churrín (Scytalopus magellanicus [Gmelin]) y el hued hued (Pteroptochos tarnii Kittlitz), pertenecientes a la familia Rhinocryptidae, se refugian bajo la quila y nidifican en troncos con cavidades cercanos al suelo (DeSanto et al. 2002, Willson et al. 2005). Otras estructuras de bosques antiguos, los grandes árboles emergentes del dosel (generalmente pioneros longevos) y árboles muertos en pie aportan sitios de anidación para especies de aves como el carpintero negro (Campephilus magellanicus [King]), el choroy (Enicognathus leptorhynchus [King]) y el rayadito (Aphrastura spinicauda, Willson et al. 1996, Cornelius 2006). En bosques jóvenes (30-80 años) con similar composición florística que bosques antiguos (> 200 años), pero que carecen de grandes árboles y sotobosque denso, el número de especies de aves disminuye de 16 a 9 y la abundancia se reduce en 60 \% (Díaz et al. 2005).

El estrato superior del bosque, el dosel, ubicado a veces por sobre 30 metros, es aún una frontera del conocimiento debido a la dificultad para acceder a él. Carecemos de datos sobre muchos procesos biológicos y químicos que ocurren en la interfase entre el bosque y la atmósfera, como las tasas de fotosíntesis, la intercepción de niebla y de nutrientes disueltos en la precipitación y la evapotranspiración desde las copas de árboles. Además el dosel alberga una gran diversidad de pequeños organismos, especialmente invertebrados, musgos y líquenes, muchos desconocidos para la ciencia (Ozzane et al. 2003, Benzing 2004, Erwin 2004). Estudios recientes (Díaz 2009) de las copas de árboles emergentes del dosel (25-30 m de altura) en bosques costeros de tipo Valdiviano, con mínima perturbación humana, cercanos a la EBSD (e.g., Gutiérrez et al. 2008b) han mostrado que un solo gran árbol emergente alberga un $30 \%$ de todas las especies de epífitas vasculares descritas para la región de bosques templados siempreverdes y un $50 \%$ de las especies chilenas conocidas la familia Himenophyllaceae (helechos película). Es decir, cada gran árbol del dosel constituye una extraordinaria reserva de diversidad. La biomasa de epífitas se relacionó positivamente con el tamaño del árbol, estimándose más de 10 toneladas de epífitas por ha (Tejo et al. 2006). Estos estudios abren numerosas interrogantes: ¿cuál es la dinámica de colonización de árboles por epífitas e invertebrados y su dependencia de la forma y edad de los árboles?, ¿qué mecanismos permiten a las enredaderas y epífitas "trasladarse" entre los árboles?, y ¿cuáles son las funciones ecológicas de la gran diversidad y biomasa de epífitas en el dosel? (Díaz 2009). Estudios en curso buscan responder las preguntas: ¿cuánto contribuye la biomasa verde de las epífitas a la productividad primaria del bosque?, y ¿cómo esta biomasa explica la diversidad de invertebrados y aves en el dosel? (Ellwood \& Foster 2004, CruzAngón \& Greenberg. 2005). Una perspectiva de largo plazo en el estudio del dosel de los bosques es necesaria para entender variaciones en la biodiversidad y productividad primaria de bosques manejados, bajo futuros escenarios de cambio en los regímenes de precipitación y temperatura.

\section{Ensambles de polinizadores en bosques de Chiloé}

Hemos estudiado durante casi una década el ensamble de polinizadores en el mosaico de bosques y praderas representado por el paisaje 
rural. La mayoría de las especies del bosque templado tienen polinizadores bióticos (SmithRamírez \& Armesto 1994, Smith-Ramírez 1993, Riveros 1991). La red de interacciones plantapolinizador del bosque del norte de Chiloé incluye 128 especies de polinizadores y 26 especies de plantas, con un total de 316 interacciones mutualistas (Smith-Ramírez et al. 2005). Los estudios se han concentrado en 11 especies de arbustos, 9 de árboles y 5 enredaderas. Los principales polinizadores identificados fueron 21 especies de himenópteros, 52 dípteros, 47 coleópteros y cuatro especies de aves. Embothrium coccineum (Proteaceae) fue la única especie polinizada por un paseriforme, Elaenia albiceps, además del picaflor, Sephanoides sephaniodes (Molina) (Smith-Ramírez \& Armesto 2003).

La red de interacciones planta-polinizador ha sido caracterizada como altamente asimétrica, ya que unas pocas especies de plantas monopolizan muchas especies de polinizadores, y un número bajo de polinizadores visitan la mayoría de las especies de plantas (Ramos-Jiliberto et al. 2009). Además la red muestra una estructura anidada, es decir, las interacciones de las especies menos conectadas son un subconjunto de las de aquellas más conectadas.

Simulaciones de pérdida de especies de la red plantas-polinizadores, que podrían ocurrir a futuro por efectos de pérdida de hábitat o explotación de bosques, muestran que si los árboles (o los parches de bosque) fuesen extraídos del paisaje rural de Chiloé esto podría resultar en una masiva extinción de plantas y animales. Esto no ocurriría si se eliminaran los arbustos, con excepción de la especie clave Tepualia stipularis (H. et A.), con 47 especies de polinizadores, cuya pérdida causaría un decrecimiento en el anidamiento de la red. La pérdida de los himenópteros de la red de polinizadores, aunque no son el grupo más diverso, produciría una extinción en cadena de polinizadores y plantas del sistema (Ramos-Jiliberto et al. 2009). Los himenóptera son el grupo de insectos más generalistas, ya que una especie (Bombus dahlbomii Guérin) realizó alrededor de un 50 \% de las visitas a flores. La extinción de esta especie afectaría la red total de interacciones.

Los estudios de polinizadores generalmente abarcan una sola temporada de floración. Sin embargo, estudios de largo plazo (3-9 años) en la EBSD de los visitantes de flores de $E$. coccineum, Amomyrtus meli, Myrceugenia planipes y M. ovata (H. et A.) Berg. var. ovata (Smith-Ramírez et al. 2005) y Eucryphia cordifolia (C. Smith, datos no publicados) en fragmentos de bosque y en árboles aislados del paisaje rural, muestran que la riqueza de especies de polinizadores y frecuencia de visitas fue variable entre años (E. cordifolia) y dependiente del tamaño del fragmento ( $E$. coccineum) (Smith-Ramírez et al. 2007). En el caso de E. cordifolia y las especies de mirtáceas hemos registrado un patrón inverso de la frecuencia de visitas de abeja europea Apis mellifera Linnaeus y el abejorro nativo $B$. dalhbomii, en años alternados. Es decir, los años que Apis es más frecuente (c. $40 \%$ de las visitas), Bombus es escaso (6\% de las visitas), y en la siguiente temporada este patrón se invierte. Entre las posibles explicaciones de este fenómeno se está investigando en estudios de largo plazo los parásitos específicos de estos himenópteros.

\section{Ensayos de restauración ecológica}

La restauración ecológica busca imitar la sucesión y acelerar la recuperación natural de un ecosistema degradado para restablecer la biodiversidad y/o los servicios ambientales. La intervención puede incluir la introducción de especies, el manejo de procesos que hayan sido alterados o de especies amenazadas, o la reversión o suspensión de los procesos de degradación. Los experimentos de restauración que hemos implementado en la EBSD son acciones que buscan restaurar especies en declinación y procesos ecológicos.

Inmersos en el paisaje rural del norte de Chiloé se encuentran pequeñas poblaciones remanentes de ciprés de las Guaitecas, Pilgerodendron uviferum (D. Don) Florín, la conífera más austral del mundo y endémica del sur de Chile y Argentina (39-55 ${ }^{\circ}$ S). En el límite norte de su distribución geográfica las poblaciones de esta especie han sido severamente reducidas y degradadas por la quema de bosques, extracción de madera, y cambio de uso de suelo. Pequeñas poblaciones de Pilgerodendron persisten en el paisaje rural con una baja diversidad genética (Allnutt et al. 2003) y comúnmente restringidas a suelos con 
drenaje pobre (Armesto et al. 1995). Bajo este escenario, decidimos realizar experimentos para identificar estrategias efectivas de restauración y promover así la recuperación de esta valiosa especie maderera en el paisaje rural. Desde el año 2002 hemos monitoreado el reestablecimiento de Pilgerodendron en áreas de matorrales sucesionales, evaluando particularmente el efecto del sustrato (musgos del género Sphagnum) y de la sombra generada por arbustos sobre el crecimiento $\mathrm{y}$ sobrevivencia de plantas establecidas de estacas provenientes de cinco poblaciones del norte de Chiloé. Las estacas fueron enraizadas y mantenidas durante dos años en el invernadero de la EBSD y luego trasplantadas a ocho parcelas experimentales de $7 \times 7 \mathrm{~m}$, distribuyendo los individuos de las cinco poblaciones en forma aleatoria entre las parcelas. Cuatro parcelas se ubicaron en un sitio con alta $(>60 \%)$ y cuatro en un sitio con baja (<10\%) cobertura del musgo Sphagnum, que reflejan mayor y menor saturación del suelo respectivamente. En cada sitio las plantas se distribuyeron regularmente, cada 1 $\mathrm{m}$, en microhábitats con y sin cobertura de arbustos. Después de cuatro años, el crecimiento de los cipreses ha sido menor en espacios abiertos. El crecimiento fue favorecido bajo la sombra de arbustos y helechos, y por bajas coberturas de Sphagnum (Fig. 6). La sobrevivencia del ciprés, independientemente de la población de procedencia, fue mayor en suelos con baja cobertura de Sphagnum y condiciones de sombra.

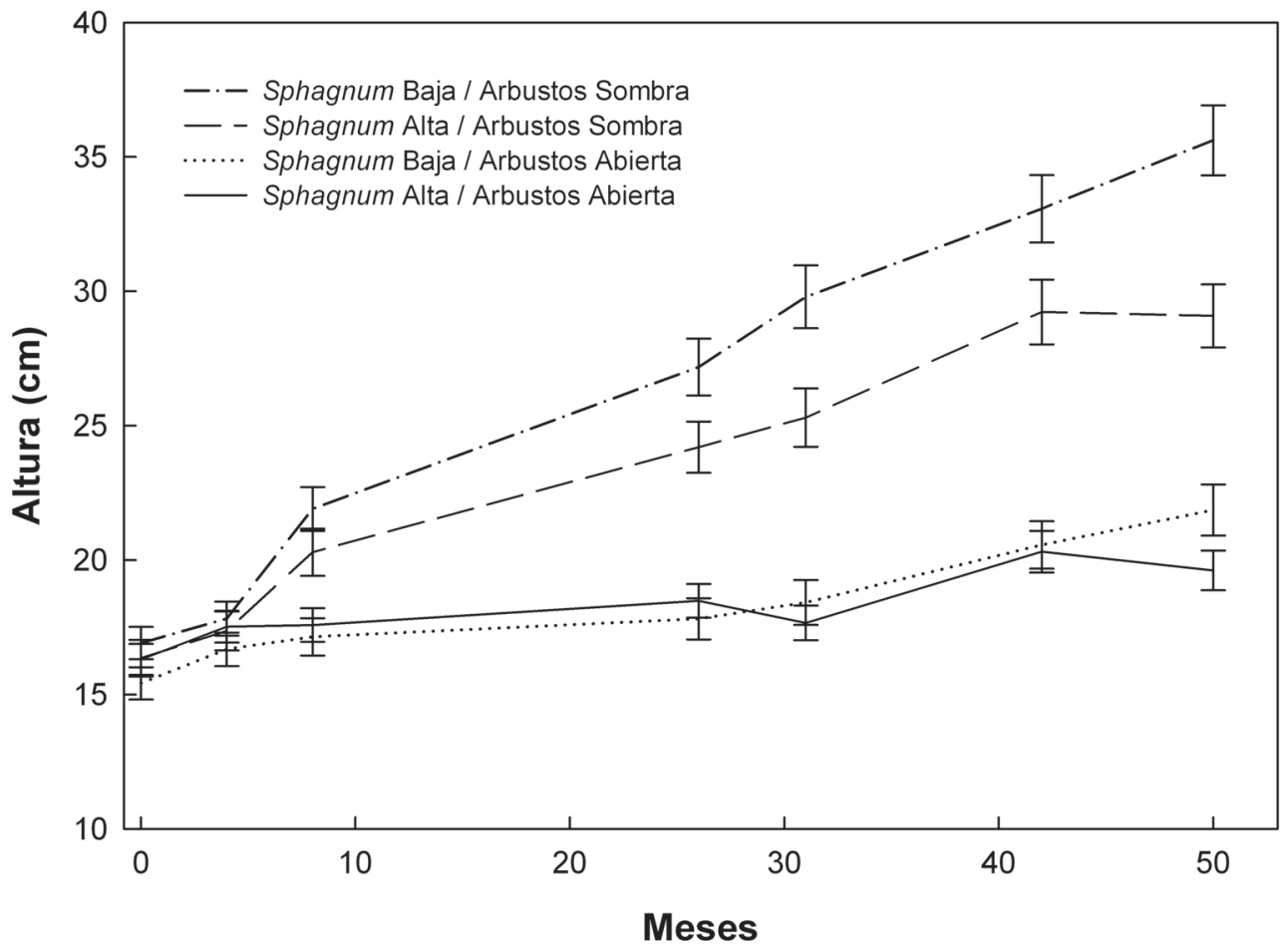

Fig. 6: Crecimiento en altura de individuos juveniles de Pilgerodendron uviferum plantados en dos sitios de matorral secundario en la EBSD bajo dos condiciones de sustrato, cobertura alta o baja de Sphagnum, y dos condiciones de cobertura de arbustos, en espacios abiertos o bajo sombra. Las plantas fueron producidas en el vivero de la EBSD a partir de esquejes de cipreses de cinco poblaciones diferentes. Las curvas corresponden al ajuste de una línea de tendencia polinomial. Se muestran los valores medios $\pm 1 \mathrm{EE}$.

Height growth of juvenile individuals of Pilgerodendron uviferum planted at two secondary shrubland sites of EBSD under two substrate conditions, high and low Sphagnum cover, and two shrub cover conditions, open area and under shrub shadow. The plants were produced within the greenhouse of EBSD from five different remaining natural populations. The curves correspond to polynomial trend fit. Mean values $\pm 1 \mathrm{SE}$ are shown. 
El mayor crecimiento de $P$. uviferum bajo sombra, a pesar de considerarse una especie colonizadora, muestra que los arbustos y helechos cumplen una importante función de "nodriza" para esta especie, facilitando su sobrevivencia y crecimiento en áreas abiertas. En contraste, altas densidades de Sphagnum, un indicador de suelos anegados, inhiben el crecimiento de $P$. uviferum en la zona de estudio. Se sugiere la restauración de poblaciones de ciprés bajo arbustos densos y en sitios con buen drenaje, como una estrategia efectiva para la recuperación de esta especie amenazada. A pesar de su crecimiento lento, su madera durable, fina y aromática es usada por artesanos y para fabricación de puertas y ventanas. Un desafío de largo plazo es evaluar los efectos de la plantación sobre la napa freática y comparar sus efectos con plantaciones de eucaliptos que han sido introducidos masivamente en áreas de cipresales quemados.

Los proyectos de restauración a nivel de una especie pueden ser infructuosos si no están acompañados de esfuerzos para mantener y reparar procesos ecológicos dañados. Estudios de sucesión en matorrales del norte de la isla han mostrado que la baja dispersión de semillas puede ser un factor limitante de la regeneración arbórea y que el establecimiento sobre detritus leñoso es mayor que sobre suelo abierto (Papic 2000). Considerando estos antecedentes, en julio de 2004 iniciamos un experimento cuyo objetivo fue determinar la posible facilitación de la regeneración arbórea en una pradera abandonada mediante el uso de perchas artificiales para atraer las aves dispersantes de semillas y la presencia de diferentes sustratos (detritus leñoso, troncos y suelo) bajo las perchas. Los resultados muestran que las perchas artificiales aumentaron varias veces la lluvia de semillas de especies arbóreas y arbustivas dispersadas por aves al área abierta (Fig. 7).

Sin embargo, a pesar de la lluvia de propágulos, el establecimiento de las especies leñosas nativas ha sido nulo en los tres tipos de sustratos durante cuatro años, a pesar de que el área se encuentra rodeada de bosques a distancias $<500 \mathrm{~m}$. En consecuencia, otros factores edáficos o ambientales limitan el establecimiento arbóreo en la pradera.
Posiblemente, es necesario considerar en el largo plazo el uso de "plantas nodrizas" y de estructuras naturales o artificiales que mejoren las condiciones de sombra e incrementen la llegada de semillas.

\section{Corredores biológicos}

En los paisajes rurales, los bosques ribereños y zonas de matorral denso conforman "corredores biológicos" que conectan parches de bosque aislados por praderas (Argent \& Zwier 2007), constituyendo vías potenciales para la dispersión activa y pasiva de plantas y animales silvestres (Johansson et al. 1996, Tewksbury et al. 2002, Haddad et al. 2003). Por esto, cabe esperar que su presencia en el paisaje incremente el desplazamiento de organismos y la conectividad de los hábitats silvestres. Sin embargo, la efectividad de los corredores como facilitadores del movimiento de animales en el paisaje antropogénico debe ser evaluada en cada caso.

Las aves del sotobosque (familias Rhinocryptidae y Furnariidae) pueden desplazarse por corredores de ancho $>25 \mathrm{~m}$ (Sieving et al. 2000). Corredores densos de vegetación tienen efectos positivos sobre la biología reproductiva del colilarga (Sylviorthorhynchus desmursii), churrín de la Mocha (Eugralla paradoxa) (Sieving et al. 2000) y chucao (ver sección anterior). El único estudio de mamíferos en corredores ribereños en la EBSD se centró en el gato guiña (Leopardus guigna [Molina]), documentando que esta especie usa quebradas con agua corriente, cubiertas por bosque denso para moverse a través del paisaje rural (Sanderson et al. 2002).

Las interacciones ecológicas también se ven modificadas en los corredores. La depredación de nidos artificiales (Willson et al. 2001) fue mayor en corredores angostos $(<10$ $\mathrm{m}$ de ancho) que en corredores anchos (> $50 \mathrm{~m}$ de ancho). Estudios recientes sobre la función ecológica de los bosques ribereños en el paisaje (Jaña-Prado 2007), comparando bosques alejados de riberas de ríos $(50-100 \mathrm{~m}$ de altitud) en la EBSD y terrenos vecinos, muestran diferencias en la lluvia de semillas dispersadas por aves frugívoras y la depredación de semillas de especies leñosas por roedores. Trampas de semillas ubicadas en 
bosques ribereños de Chiloé recibieron un número de semillas superior en un orden de magnitud al colectado en los márgenes o interior de bosques no ribereños (Fig. 8), al mismo tiempo que el porcentaje de depredación de semillas fue menor en comparación a otros hábitats.

La alta cantidad de semillas de especies dispersadas por aves en bosques ribereños sugiere que las aves frugívoras usan más frecuentemente estos hábitats. Los bosques de las riberas contribuirían a mantener la conectividad del paisaje rural para aves frugívoras y plantas cuyos frutos son consumidos por las aves. Asimismo, los árboles ribereños, que reciben más luz que en el interior del bosque podrían actuar como atrayentes de las aves y fuentes de propágulos para el resto del mosaico rural (Jaña-Prado 2007).

\section{PROGRAMA DE EDUCACIÓN ECOLÓGICA E INSERCIÓN EN EL ENTORNO RURAL}

El conocimiento generado por las investigaciones científicas realizadas en la EBSD ha sido difundido directamente a usuarios de la comunidad local y regional, sin ningún tipo de "intermediario" y en forma casi inmediata. Esta es una fortaleza propia de los sitios de estudios ecológicos de largo plazo.

La metodología denominada "ciclo de indagación de primera mano" (Rozzi et al. 2000, Feinsinger 2003, Papic \& Armesto 2005), canalizada a través de talleres de Enseñanza de la Ecología en el Patio de la Escuela (EEPE) se practica en escuelas rurales de la Isla Grande de Chiloé desde el año 1997, promovida por el programa de educación de la EBSD. Dicha metodología se basa en la capacitación directa de profesores, alumnos,

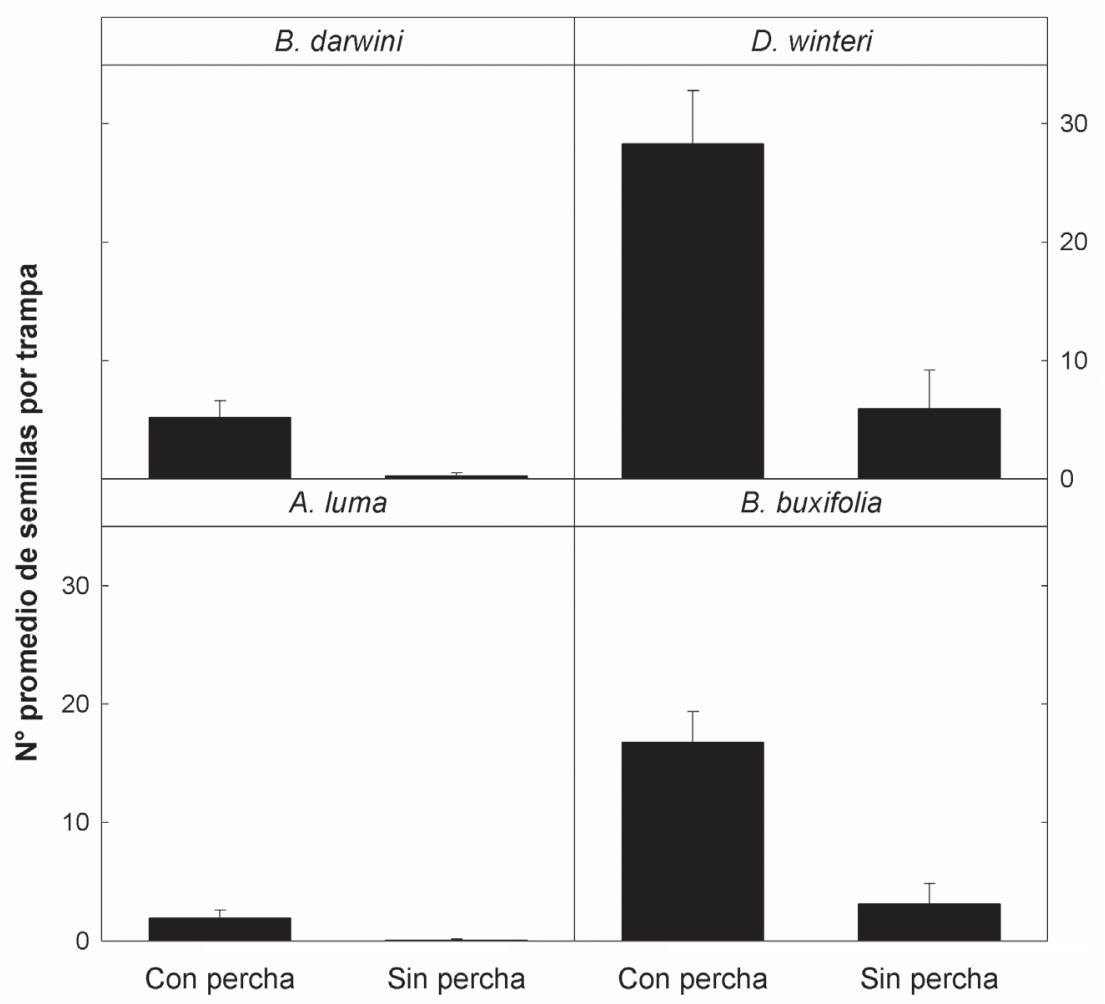

Fig. 7: Número promedio de semillas por trampa de cuatro especies leñosas (Drimys winteri, Amomyrtus luma, Berberis buxifolia, Berberis darwini) registradas durante la temporada de muestreo (primavera-verano) del año 2005 en trampas de semillas bajo perchas artificiales $(\mathrm{n}=15)$ y trampas sin perchas $(n=15)$ instaladas en una pradera de la EBSD. Barras de error: \pm 1 EE.

Average number of seeds per trap of four woody species recorded during the sampling period (Spring-Summer) of year 2005 within traps under artificial perches $(n=15)$ and traps without perches $(n=15)$ installed in a abandoned pasture of EBSD. Error bars: $\pm 1 \mathrm{SE}$. 
guardaparques y propietarios para responder preguntas simples sobre su entorno usando el método científico. El método requiere que los participantes en los talleres hagan sus preguntas y lleven a cabo las observaciones y experimentos necesarios para responderlas, además de presentar al grupo de trabajo sus conclusiones y reflexiones. Desde el año 2000 a la fecha, la EBSD ha dictado talleres para más de 500 escolares y 60 docentes de Educación Básica de Chiloé, que se han extendido también a otros puntos del país (Puerto Montt, Valdivia, Santiago, La Serena). A su vez, los participantes de los talleres han replicado la metodología de indagación de primera mano con sus estudiantes en sus establecimientos educacionales, sumando un efecto multiplicativo.

El ciclo de indagación es también una herramienta didáctica para apoyar la conservación e investigación ecológica en áreas naturales protegidas, privadas o públicas, a través del trabajo con los guardaparques.
Personal de CONAF ha participado en talleres dedicados específicamente al diseño y realización de estudios sobre biodiversidad y el desarrollo de senderos de instrucción para los visitantes. Los talleres permiten también el diálogo entre científicos y estudiantes de centros académicos asociados a la EBSD y comunidades locales, promoviendo las indagaciones en colaboración, y el análisis de los problemas de interés para las comunidades de guardaparques y maestros, que pueden estimular estudios de más largo plazo.

El bajo costo de los talleres, el uso de recursos locales, evitando el empleo de herramientas sofisticadas, inaccesibles a escuelas o parques, en la investigación y la comunicación pública de los resultados y reflexiones que derivan de las indagaciones realizadas, hacen de esta metodología un medio ideal para formar una comunidad creadora de conocimientos y con capacidad crítica respecto de su entorno ambiental y social.

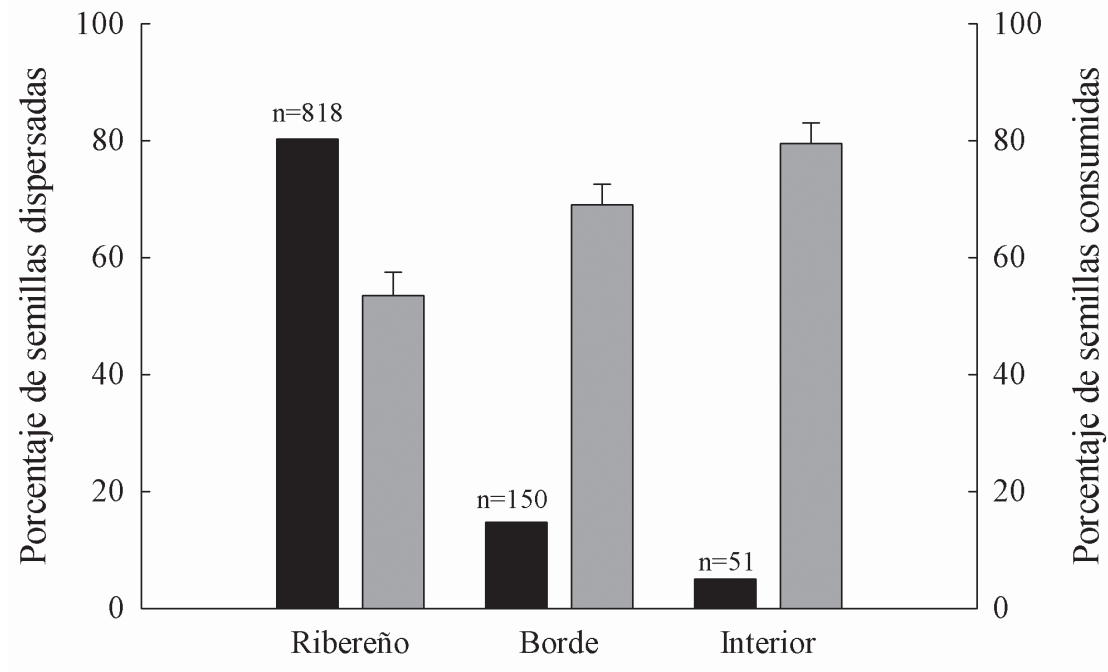

Hábitat

Fig. 8: Porcentaje de semillas dispersadas en hábitats ribereños y no ribereños (borde e interior), en relación al total de semillas caídas en trampas (eje izquierdo, barras negras). Diferencia entre hábitats: $\chi^{2}=1,025, \mathrm{df}=2, \mathrm{P}<0.001$. También se presenta el porcentaje promedio de remoción de semillas por depredadores de Embothrium coccineum y Aristotelia chilensis (eje derecho, barras grises) en los diferentes hábitats. Diferencia entre medias: $\mathrm{t}_{\text {interior-ribereño }}=4.89 ; \mathrm{P}<0.001, \mathrm{t}_{\text {borde- }}$ ribereño $=2.91 ; \mathrm{P}<0.05, \mathrm{t}_{\text {interior-borde }}=2.12 ; \mathrm{P}<0.05$ (después de corrección por Bonferroni).

Percentage of dispersed seeds within riparian and non-riparian habitats (border and interior of forest), in relation to the total number of fallen seeds in the traps (left axis, black bars). Differences between habitats: $\chi 2=1,025$, df $=2$, $\mathrm{P}<0.001$. Mean percentage of seed removal by predators of Embothrium coccineum and Aristotelia chilensis (right axis, grey bars) within each habitat is also shown. Differences between means: $\mathrm{t}_{\text {interior-riparian }}=4.89 ; \mathrm{P}<0.001$, $\mathrm{t}$ edgeriparian $=2.91 ; \mathrm{P}<0.05, \mathrm{t}_{\text {interior-edge }}=2.12 ; \mathrm{P}<0.05$ (after Bonferroni correction). 
La metodología EEPE, también ha sido aplicada para difundir la ecología de ecosistemas. Junto a un grupo de 60 alumnos de tres escuelas rurales de la comuna de Ancud, se investigó durante el año escolar 2006 el proceso de descomposición de la materia orgánica, con la finalidad de que niños y niñas comprendieran sus bases biogeoquímicas y su relación con el crecimiento de las plantas, visualizando el proceso directamente en el patio de sus escuelas. En cada escuela se instalaron ensayos de descomposición (método de bolsa de malla) con hojas de dos especies leñosas nativas (Aristotelia chilensis Mol. "Maqui" y Nothofagus nitida “Coiguie de Chiloé”) y dos sustratos orgánicos de origen antropogénico (papel blanco y madera de pino). Además se montaron experimentos en invernadero de cultivo de especies leñosas nativas en diferentes medios. Los sustratos orgánicos presentaron distintas tasas de degradación durante el periodo de ensayo (seis meses), donde las hojas de especies nativas perdieron mayor masa que los otros materiales (Fig. 9). Los resultados documentaron indirectamente la acción de diferentes tipos de organismos descomponedores y fueron presentados en un Congreso Científico Escolar realizado en la EBSD.

En forma paralela a la EEPE, las especies carismáticas de animales (amenazados o ecológicamente menos conocidos) presentes en la EBSD y sus alrededores han servido como pilares centrales de talleres de educación y materiales de difusión dirigidos a informar y crear interés en la comunidad local y nacional. Por ejemplo, el marsupial monito del monte, ha sido el objeto de programas de televisión local y nacional y reportajes de prensa. Mayor información se difunde por internet, afiches y charlas para colegios, organizaciones locales y gubernamentales. El programa de educación de la EBSD ha difundido los estudios sobre el zorro de Darwin (L. fulvipes) y el pudú (Pudu pudu [Molina]), dentro de un programa conjunto con organizaciones locales que promueven su protección, la tenencia responsable y esterilización de mascotas en zonas rurales.

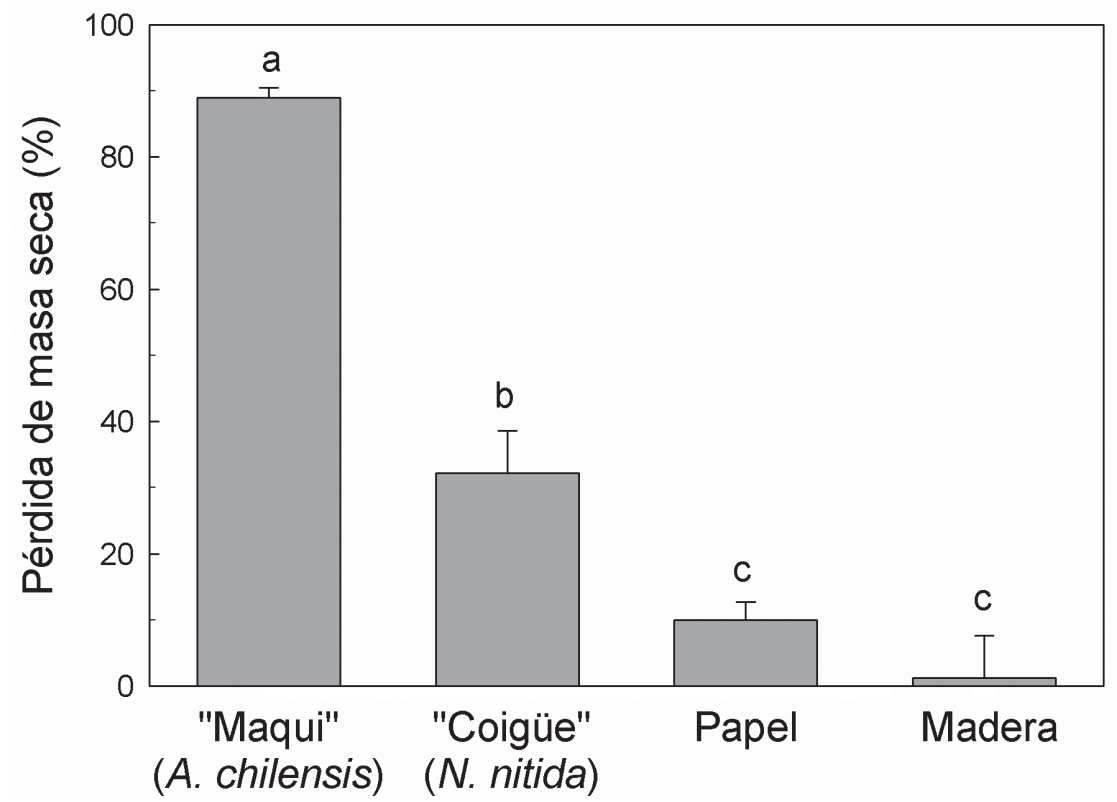

Fig. 9: Degradación (pérdida de masa) de hojas de dos árboles nativos (Aristotelia chilensis y Nothofagus nitida), papel y madera de pino durante seis meses en los patios de escuelas rurales de la comuna de Ancud $(\mathrm{N}=3)$. Las letras distintas indican diferencias significativas $(\mathrm{P}<0.05)$ entre los distintos tipos de material orgánico en descomposición de acuerdo a un ANDEVA.

Degradation (mass loss) of leaves of two native trees (Aristotelia chilensis y Nothofagus nitida), paper and pine wood during six moths in rural schoolyards of Ancud district $(\mathrm{N}=3)$. Different letters shows significant differences $(\mathrm{P}<0.05)$ between decomposing organic materials according to ANOVA. 


\section{Vínculos con la comunidad local}

Uno de los objetivos de la EBSD es establecer mecanismos efectivos de comunicación entre ecólogos y otros profesionales con la comunidad local. Parte de la infraestructura desarrollada en la EBSD con este objetivo es el Centro de Educación Ambiental "Beagle" y el Sendero de Interpretación "Pichihuillilemu" (pequeño bosque del Sur). El "Beagle" ha permitido la realización de talleres y cursos con público local. Su arquitectura y ubicación junto al bosque ribereño (Fig. 1) estimula el trabajo creativo y la curiosidad por el entorno. De la misma forma, el "Pichihuillilemu" recibe la visita de más de 100 escolares y numerosos turistas cada año, quienes aprenden sobre la flora, fauna y ecología del bosque chilote. Algunos talleres sobre temas específicos, tales como la aplicación del conocimiento científico a la propagación de especies nativas (usando el Vivero "Luis Cavieres") y manejo amigable del bosque nativo para conservar la biodiversidad, han atraído mucho púbico de la comunidad rural.

Otras vías de comunicación del conocimiento generado en la EBSD al público no científico son artículos en la prensa local (diarios La Estrella de Chiloé y El Insular), el Boletín de la EEPE (Audubon Society) y la televisión local. Por ejemplo, el proyecto de conservación del zorro de Darwin permitió la creación, junto a la productora nacional Cábala, de 16 microprogramas sobre el estudio de esta especie emblemática. Además la EBSD se ha hecho el propósito de estar presente regularmente en Muestras Costumbristas anuales de la comuna de Ancud, principal celebración de la comunidad chilota, donde se combinan la cultura y tradiciones con el aprovechamiento de la biodiversidad. El trabajo con la comunidad ha permitido transferir el conocimiento directamente a los pequeños propietarios y habitantes rurales (Tabla 3). La inefectividad en el traspaso del conocimiento científico a los que toman las decisiones o aplican esta información constituye una de las mayores causas de subdesarrollo y de la pérdida de capital natural. Para reparar parte de esta deficiencia, la EBSD mantiene un programa de educación permanente, liderado directamente por investigadores (estudiantes universitarios de pre y postgrado y profesionales), en coordinación con otros sitios dentro del Instituto de Ecología y Biodiversidad (IEB).

\section{Proyecto agroproductivo}

Desde su creación, un objetivo de la EBSD ha sido desarrollar actividades productivas propias del entorno rural del norte de Chiloé, aprovechando los terrenos y en conformidad con el modo de vida local. A partir del año 2008 estamos desarrollando un nuevo proyecto agroproductivo que tiene por principal objetivo convertir a la EBSD en un modelo de producción agrícola y forestal sustentable, integrando la conservación de la biodiversidad, en el norte de la Isla Grande de Chiloé. Este modelo se basa en el desarrollo de actividades económicas innovadoras y de baja inversión, que esperamos puedan replicarse en otras áreas rurales del sur de Chile, especialmente con pequeños propietarios. Un elemento esencial de este trabajo es la cooperación entre la EBSD y propietarios vecinos, en combinación con talleres de capacitación científica y técnica para el manejo de especies tradicionales y nuevas, que aprovechen el potencial productivo de la zona.

La producción actual de la EBSD se basa en el ganado ovino y esquila de lana, cultivo de papas nativas y ajos chilotes, cosecha de manzanas para la producción de chicha $y$ vinagre (a pequeña escala), además de la producción de plantas nativas de vivero. Parte de la producción es para consumo interno $\mathrm{y}$ parte se comercializa. Nuestro plan es diversificar y aumentar progresivamente la producción dentro de las superficies destinadas para este fin en el plan de manejo del predio. Entre las nuevas propuestas está un "huerto experimental" mixto de especies arbóreas nativas que generarán una variedad de productos forestales no madereros y madereros, basados en especies de valor tradicional como Gevuina avellana Mol., Amomyrtus luma y Embothrium coccineum. Estas especies de origen en la EBSD se han propagado en nuestro vivero. En colaboración con el programa de Artesanías de la Pontificia Universidad Católica de Chile participamos en un estudio sobre fibras vegetales utilizadas tradicionalmente por comunidades de Chiloé. Este proyecto complementario a los estudios 
socioecológicos de largo plazo de la EBSD, permitirá la aplicación de información científica por la comunidad, a corto, mediano y largo plazo.

\section{CONCLUSIÓN Y PROYECCIONES}

El paisaje rural del norte de Chiloé, donde se encuentra la EBSD, ofrece oportunidades para integrar el conocimiento y uso de recursos biológicos a través de un gradiente completo desde bosques antiguos, con árboles de más de tres siglos, y escasa perturbación antrópica, hasta ambientes dependientes de la acción humana, como praderas, cultivos agrícolas y plantaciones forestales. En la matriz seminatural del paisaje rural coexisten distintos usos del suelo, parches de bosques nativos, praderas y matorrales en diferentes estados sucesionales, creando un espacio de convivencia humana con las plantas y animales nativos. Además, la EBSD es parte de un ambiente rural donde el estilo de vida tradicional del habitante chilote se mezcla con el rápido y cambiante mundo globalizado. En este contexto, la investigación ecológica de largo plazo es relevante para entender cómo los cambios en los ecosistemas terrestres, determinados por la creciente demanda global por recursos, la expansión de especies introducidas y de nuevos cultivos (e.g., salmón) y nuestras prácticas de manejo, afectarán la biodiversidad y otras funciones ecológicas de importancia regional y global.

Dos ejemplos de servicios de los ecosistemas a la sociedad que estamos investigando son: (1) la productividad primaria (incluyendo la fijación y retención de carbono) en bosques antiguos y sucesionales, y (2) la sustentabilidad del ciclo hidrológico en zonas rurales, donde son afectados directamente por la tala de bosques, la explotación y drenaje de humedales, y las plantaciones forestales. Tales estudios requieren un enfoque ecosistémico, experimental e investigación ecológica de largo plazo. Esta información es esencial para proponer prácticas de manejo sustentable y uso del paisaje a escala local. Los cambios globales, incluyendo el cambio climático, afectarán las culturas locales, a través de la pérdida de diversidad de especies propias e introducción de especies exóticas, homogenización de paisajes y pérdida de recursos tradicionales. Estudios ecológico-sociales de largo plazo nos sirven para idear estrategias que permitan mantener un flujo sostenido de bienes y servicios de los ecosistemas, reduciendo las externalidades y maximizando los beneficios para la sociedad regional. Por ejemplo, la conservación de los bosques para la producción de agua para el consumo humano tiene un valor económico favorable para la sociedad (The Economist, January 2005). El tema de la capacidad de recuperación (o resiliencia) de los ecosistemas nativos después de catástrofes naturales como incendios o sequías es relevante ante un escenario de cambio climático, que predice una disminución de las precipitaciones en Chile sur-central. Los estudios de largo plazo nos permiten obtener datos experimentales sobre los efectos de tales cambios. Un valor agregado de los bosques templados y subantárticos es su carácter de fronteras ante el avance humano en el planeta (Mittermeier et al. 2003), especialmente debido a la ausencia de polución atmosférica, que domina en ecosistemas templados del hemisferio norte. Los bosques chilenos (y sus sistemas fluviales asociados) constituyen modelos de la condición preindustrial de los ciclos de nutrientes (Hedin et al. 1995, Perakis \& Hedin 2002, Armesto et al. 2009). Estudios de largo plazo nos permiten anticipar los cambios en los procesos biogeoquímicos que sustentan los ecosistemas ante el avance de la agricultura y la industrialización.

Un aporte innovador de la red chilena ESELP es la mantención de registros fenológicos y reproductivos de numerosas especies de plantas y animales nativos que habitan los ambientes rurales. Estos registros simples y de bajo costo son relevantes para detectar, prevenir o mitigar los efectos del acelerado cambio del paisaje (Echeverría et al. 2007), y anticipar los cambios climáticos esperados en la región. En este sentido, investigaciones sistemáticas, censos periódicos y modelos demográficos de especies focales de fauna de diverso origen y carácter, pero sensibles a cambios del paisaje, como son el rayadito, el chucao y/o el monito del monte, pueden aportar información valiosa sobre la persistencia de la biodiversidad en el paisaje rural, bajo diferentes escenarios reales o simulados de manejo, cambios de cobertura 
y alteraciones del clima. Nuestro aprendizaje en el paisaje chilote puede enseñarnos cómo enfrentar exitosamente el futuro.

\section{MATERIAL COMPLEMENTARIO}

La versión en inglés de este artículo está disponible como Material Complementario online en http://rchn.biologiachile.cl/ suppmat/2010/1/MC_Carmona_et_al_2010.pdf

\section{AGRADECIMIENTOS}

Agradecemos a quienes contribuyeron a adquirir parte de los terrenos que hoy conforman la EBSD (en orden alfabético): J. Bacigalupo, L. Cavieres, F. Jaksic, H. Jiménez, P. León, H. Núñez, A. Peñaloza, C. Villagrán, J. Yáñez. También agradecemos el generoso apoyo en diversas etapas del proyecto de: $\mathrm{O}$. Barbosa, I. Casassa, T. Castellón, C. Cornelius, E. del Val, E. Elgueta, P. Feinsinger, A. Garrido, J. Hertz, J. G. Kenagy, J. Larraín, I. Lazzoni, A. Magrach, E. Mancilla, P. Marín, S. Merino, A. Mora, J. Moreno, P. Naulin, T. Naulin, M. Piazzón, F. Pugnaire, R. Quispe, R. Riveros, A. Rodríguez, M. Rodríguez-Gironés, C. Sabag, J. Sanderson, L. Santamaría, K. E. Sieving, F. Valladares, A. Vidal, C. Villavicencio, W. van Dongen, H. Yáñez, G. Zegers, R. Zúñiga y varias generaciones de estudiantes y docentes del curso Ecología de Bosques. Agradecemos el compromiso y dedicación de Juan Vidal (encargado del vivero) y Yuri Zúñiga (actual guardaparque de la EBSD). Finalmente, agradecemos a las siguientes fuentes de financiamiento: Fondo de las Américas (Chile), Darwin Initiative (UK), Cátedra Presidencial en Ciencias (a JJA), Proyectos Fondecyt 1020550, 1050225, 1060186, 1090794, 11085007, 3070011, Fondecyt-FONDAP 1501-0001 (a CASEB, Pontificia Universidad Católica de Chile), INCO-DC program of the European Union (Biocores y Reforlan), Becas doctorales CONICYT, ICM-P05-002 (Mideplan) al IEBChile, PBF-23-CONICYT al IEB-Chile, US National Science Foundation (a MFW), DAAD (a AGG), Gary Machlis and The Cannon National Parks Science Scholars Program (a IAD), CIPMA-GEF (2003), National Geographic Society, Fundación COPEC, CORFO-Innova,
Universidad Santo Tomás, Departamento de Geofísica, Universidad de Chile, Fundación BBVA (BIOCON06/109 y 110), EXPLORAConicyt y Bosque Modelo Chiloé. Agradecemos también el apoyo permanente de CONAMA, CONAF, SAG, X Región y la comunidad chilota.

\section{LITERATURA CITADA}

ALLNUTT TR, AC NEWTON, AC PREMOLI \& A LARA (2003) Genetic variation in the threatened South American conifer Pilgerodendron uviferum (Cupressaceae), detected using RAPD markers. Biological Conservation 114: 245-253

ARAVENA JC, MR CARMONA, CA PÉREZ \& JJ ARMESTO (2002) Changes in tree species richness, stand structure and soil properties in a successional chronosequence in northern Chiloé Island, Chile. Revista Chilena de Historia Natural 75: 339-360.

ARGENT DG \& RJ ZWIER (2007) Seasonal use of recently fenced agricultural riparian habitat by avifauna in Pennsylvania. Northeastern Naturalist 14: 361-374.

ARMESTO JJ (1990) Long-term studies - a research priority for today's ecology. Revista Chilena de Historia Natural 63: 7-9.

ARMESTO JJ \& R ROZZI (1989) Seed dispersal syndromes in the rain forest of Chiloé: Evidence for the importance of biotic dispersal in a temperate rain forest. Journal of Biogeography 16: 219-226.

ARMESTO JJ, I DÍAZ, C PAPIC \& MF WILLSON (2001) Seed rain of fleshy and dry propagules in different habitats in the temperate rainforests of Chiloé Island, Chile. Austral Ecology 26: 311-320.

ARMESTO JJ, C VILLAGRÁN, JC ARAVENA, CA PÉREZ, C SMITH-RAMÍREZ, M CORTÉS \& LO HEDIN (1995) Conifer forests of the Chilean coastal range. En: Hill RS \& N Enright (eds) Ecology of the southern conifers: 156-170. Melbourne University Press, Melbourne.

ARMESTO JJ, C VILLAGRÁN \& MK ARROYO (eds) (1996) Ecología de los bosques nativos de Chile. Editorial Universitaria, Santiago.

ARMESTO JJ, R ROZZI, C SMITH-RAMÍREZ \& MTK ARROYO (1998) Conservation targets in South American temperate forests. Science 282: 12711272.

ARMESTO JJ, S BAUTISTA, E DEL VAL, B FERGUSON, X GARCÍA et al. (2007) Towards an ecological restoration network: Reversing land degradation in Latin America. Frontiers in Ecology and the Environment 5: W1-W4.

ARMESTO JJ, MR CARMONA, JL CELIS-DIEZ, I DÍAZ, A GAXIOLA et al. (2009) Old-growth temperate rain forests of South America: Conservation status, plant-animal interactions and baseline biogeochemical processes. En: Wirth C, G Gleixner \& M Heimann (eds) Old growth forests: Function and fate of a vanishing ecosystem type: 376-390. Ecological Studies, Vol. 207, Springer Verlag.

ARROYO MK, JJ ARMESTO, R ROZZI \& A PEÑALOZA (1999) Bases de la sustentabilidad ecológica y sus implicaciones para el manejo y conservación del bosque nativo en Chile. En: Donoso C \& A 
Lara (eds) Silvicultura de los bosques nativos de Chile: 35-60. Editorial Universitaria, Santiago, Chile.

BENZING DH (2004) Vascular epiphytes. En: Lowman MD \& HB Rinker (eds) Forest canopies: 175-211. Elsevier Academic Press, Massachusetts, USA.

BOZINOVIC F, G RUIZ \& M ROSENMANN (2004) Energetics and torpor of a South American living fossil, the microbiotheriid Dromiciops gliroides. Journal of Comparative Physiology B 174: 293-297.

CARMONA MR (2004) Fijación no simbiótica de nitrógeno en la detritósfera de un bosque templado en Chiloé: Regulación interna y su relación con el proceso de descomposición. Tesis de Doctorado, Facultad de Ciencias, Universidad de Chile, Santiago.

CARMONA MR, JJ ARMESTO, JC ARAVENA \& CA PÉREZ (2002) Coarse woody debris biomass in successional and primary temperate forests in Chiloé Island, Chile. Forest Ecology and Management 164: 265-275.

CASTELLÓN TD \& KE SIEVING (2006a) An experimental test of matrix permeability and corridor use by an endemic understory bird. Conservation Biology 20: 135-145.

CASTELLÓN TD \& KE SIEVING (2006b) Landscape history and fragmentation effects on patch occupancy: Empirical models for an endemic forest bird with limited dispersal ability. Ecological Applications 16: 2223-2234.

CASTELLÓN TD \& KE SIEVING (2007) Patch network criteria for dispersal-limited endemic birds of South American temperate rain forest. Ecological Applications 17: 2152-2163.

CELIS-DIEZ JL (2010) Historia natural y demografía del marsupial arbóreo Dromiciops gliroides en bosques nativos primarios y degradados del norte de la isla grande de Chiloé. Tesis de Doctorado, Facultad de Ciencias, Universidad de Chile, Santiago.

CHRISTIE DA \& JJ ARMESTO (2003) Regeneration microsites and tree species coexistence in temperate rain forests of Chiloé Island, Chile. Journal of Ecology 91: 776-784.

CLARK JS, B BECKAGE, P CAMILL, B CLEVELAND, J HILLERISLAMBERS et al. (1999) Interpreting recruitment limitation in forests. American Journal of Botany 86: 1-16.

CLYMO RS \& PM HAYWARD (1982) The ecology of Sphagnum. En: Smith AJE (ed) Bryophyte ecology: 229-289. Chapman and Hall, London New York.

CLYMO RS, J TURUNEN \& K TOLONEN (1998) Carbon accumulation in peatland. Oikos 81: 368388.

COFRÉ H \& PA MARQUET (1998) Conservation status, rarity, and geographic priorities for conservation of Chilean mammals: An assessment. Biological Conservation 88: 53-68.

CORNELIUS C (2006) Genetic and demographic consequences of human-driven landscape changes on bird populations: The case of Aphrastura spinicauda (Furnariidae) in the temperate rainforest of South America. Ph. D. Dissertation, University of Missouri, St. Louis.

CORNELIUS C (2008) Spatial variation in nest-site selection by a secondary cavity-nesting bird in a human-altered landscape. The Condor 110: 615626.

CORNELIUS C, H COFRÉ, \& PA MARQUET (2000)
Effects of habitat fragmentation on bird species in a relict temperate forest in semiarid Chile. Conservation Biology 14: 534-543.

CRUZ-ANGÓN A \& R GREENBERG (2005) Are epiphytes important for birds in coffee plantations? An experimental assessment. Journal of Applied Ecology 42: 150-159.

DE SANTO TL, MF WILLSON, KE SIEVING \& JJ ARMESTO (2002) Nesting biology of tapaculos (Rhinocryptidae) in fragmented south-temperate rainforests in Chile. Condor 104: 482-495.

DGF \& CONAMA (2006) Estudio de la variabilidad climática en Chile para el siglo XXI. Informe Final. Departamento de Geofísica, Facultad de Ciencias Físicas y Matemáticas, Universidad de Chile. Comisión Nacional del Medio Ambiente, Santiago, Chile.

DÍAZ IA (2009) Linking composition, structure and functions of biodiversity: Relationships among epiphytes, invertebrates and birds in the canopy of Chilean temperate rainforests. $\mathrm{Ph}$. D. Dissertation, University of Florida, Gainesville.

DÍAZ I, JJ ARMESTO, S REID, KE SIEVING \& MF WILLSON (2005) Linking forest structure and composition: Avian diversity in successional forests of Chiloé Island, Chile. Biological Conservation 123: 91-101.

DÍAZ IA, JJ ARMESTO \& MF WILLSON (2006) Mating success of the endemic Des Murs' Wiretail (Sylviorthorhynchus desmursii, Furnariidae) in Chilean fragmented rainforests. Austral Ecology 31: $13-21$.

DÍAZ MF (2004) Limitantes biológicas e hidrológicas de la sucesión secundaria en bosques de Chiloé. Tesis de Doctorado, Facultad de Ciencias, Universidad de Chile, Santiago.

DÍAZ MF (2008) Guía práctica de terreno para realizar un manejo sustentable del pompón (Sphagnum magellanicum). URL: http://www.sendadarwin.cl/ espanol/wp-content/uploads/2009/12/ guia_de_terreno_ponpon.pdf (accedido Octubre $31,2008)$.

DÍAZ MF \& JJ ARMESTO (2007) Physical and biotic constraints on tree regeneration in secondary shrublands of Chiloe Island, Chile. Revista Chilena de Historia Natural 80: 13-26.

DÍAZ MF, S BIGELOW \& JJ ARMESTO (2007) Alteration of the hydrologic cycle due to forest clearing and its consequences for rainforest succession. Forest Ecology and Management 244: 32-40.

DÍAZ MF, J LARRAÍN, G ZEGERS \& C TAPIA (2008) Caracterización florística e hidrológica de turberas de la Isla Grande de Chiloé, Chile. Revista Chilena de Historia Natural 81: 455-468.

DÍAZ V (2005) Evaluación de la dimensión humana, a través del estudio de las actitudes y conocimientos de la gente de la isla Grande de Chiloé, X Región, para futuros planes de conservación de fauna silvestre y su hábitat. Tesis de Pregrado, Universidad Católica de Temuco, Temuco, Chile.

ECHEVERRIA C, AC NEWTON, A LARA, JMR BENAYAS \& DA COOMES (2007) Impacts of forest fragmentation on species composition and forest structure in the temperate landscape of southern Chile. Global Ecology and Biogeography 16: 426-439.

ELLWOOD MDF \& WA FOSTER (2004) Doubling the estimate of invertebrate biomass in a rainforest canopy. Nature 429: 549-551. 
ERWIN TL (2004) The biodiversity question: How many species of terrestrial arthropods are there? En: Lowman MF \& HB Rinker (eds) Forest Canopies: 259-269. Elsevier, Burlington.

FATTORINI M \& S HALLE (2004) The dynamic environmental filter model: How do filtering effects changes in assembling communities after disturbace? En: Temperton VM, RJ Hobbs, T Nuttle \& S Halle (eds) Assembly rules and restoration ecology: Bridging the gap between theory and practice: 96-114. Island Press, Washington.

FEINSINGER P (2003) El diseño de estudios de campo para la conservación de la biodiversidad. Ed. FAN, Santa Cruz de la Sierra, Bolivia.

FOSTER SA (1999) The geography of behaviour: An evolutionary perspective. Trends in Ecology \& Evolution 14: 190-195.

FRANKLIN JF, K CROMACK JR, W DENISON, A MCKEE, C MASER, J SEDELL, F SWANSON \& G JUDAY (1981) Ecological characteristics of old-growth Douglas-fir forests. USDA Forest Service General Technical Report PNW-111.

GARREAUD R \& D BATTISTI (1999) Interannual (ENSO) and interdecadal (ENSO-like) variability in the southern hemisphere tropospheric circulation. Journal of Climate 12: 2113-2123.

GONZÁLEZ-ACUÑA D, C BRICEÑO, A CICCHINO, SM FUNK \& JE JIMÉNEZ (2007) First records of Trichodectes canis (Insecta: Phthiraptera) from Darwin's fox, Pseudalopex fulvipes (Mammalia: Carnivora: Canidae). European Journal of Wildlife Research 53: 76-79.

GORHAM E (1991) Northern peatlands - role in the Carbon-cycle and probable responses to climatic warming. Ecological Applications 1: 182-195.

GRIGERA D (1982) Ecología alimentaria de algunas passeriformes insectívoras frecuentes en los alrededores de S. C. de Bariloche. Ecología (Argentina) 7: 67-84

GUTIÉRREZ AG, JJ ARMESTO \& JC ARAVENA (2004) Disturbance and regeneration dynamics of an old-growth North Patagonian rain forest in Chiloé Island, Chile. Journal of Ecology 92: 598608.

GUTIÉRREZ AG, O BARBOSA, DA CHRISTIE, E DELVAL, HA EWING, et al. (2008a) Regeneration patterns and persistence of the fog-dependent Fray Jorge forest in semiarid Chile during the past two centuries. Global Change Biology 14: 161-176.

GUTIÉRREZ AG, JC ARAVENA NV, CARRASCOFARÍAS, DA CHRISTIE, M FUENTES \& JJ ARMESTO (2008b) Gap-phase dynamics and coexistence of a long-lived pioneer and shadetolerant tree species in the canopy of an oldgrowth coastal temperate rain forest of Chiloé Island, Chile. Journal of Biogeography 35: 16741687.

GUTIÉRREZ AG, JJ ARMESTO, JC ARAVENA, NV CARRASCO, DA CHRISTIE et al. (2009) Structural and environmental characterization of old-growth temperate rainforests of northern Chiloé Island, Chile: Regional and global relevance. Forest Ecology and Management 258: 376-388.

HADDAD NM, DR BOWNE, A CUNNINGHAM, BJ DANIELSON, DJ LEVEY, S SARGENT \& T SPIRA (2003) Corridor use by diverse taxa. Ecology 84: 609-615.
HEDIN LO, JJ ARMESTO \& AH JOHNSON (1995) Patterns of nutrient loss from unpolluted, oldgrowth temperate forests: Evaluation of biogeochemical theory. Ecology 76: 493-509.

HENDRICKSON O (2003) Old-growth forests: Data gaps and challenges. Forestry Chronicle 79: 645651.

HERSKHOVITZ P (1999) Dromiciops gliroides Thomas 1894, last of the Microbiotheria (Marsupialia), with a review of the family Microbiotheriidae. Fieldiana Zoology 93: 1-60.

HIMES CMT, MH GALLARDO \& JG KENAGY (2008) Historical biogeography and post-glacial recolonization of South American temperate rain forest by the relictual marsupial Dromiciops gliroides. Journal of Biogeography 35: 1415-1424.

IPPI S \& A TREJO (2003) Dinámica y estructura de bandadas mixtas de aves en un bosque de lenga (Nothofagus pumilio) del noroeste de la Patagonia argentina. Ornitologia Netropical 14: 353-362.

IRESTEDT M, US JOHANSSON, TJ PARSONS \& PGP ERICSON (2001) Phylogeny of major lineages of suboscines (Passeriformes) analysed by nuclear DNA sequence data. Journal of Avian Biology 32: 15-25.

JAÑA-PRADO RC, JL CELIS-DIEZ, AG GUTIÉRREZ, C CORNELIUS \& JJ ARMESTO (2006) Diversidad en bosques fragmentados de Chiloé: ¿Son todos los fragmentos iguales? En: Grez AA, JA Simonetti \& RO Bustamante (eds) Biodiversidad en ambientes fragmentados de Chile: Patrones y procesos a diferentes escalas: 159-190. Editorial Universitaria, Santiago, Chile.

JAÑA-PRADO RC (2007) Seed rain of bird-dispersed species in riparian and upland forests in a rural landscape of Northern Chiloe Island, Chile. MSc. Thesis, Facultad de Ciencias, Universidad de Chile, Santiago.

JARAMILLO A (2003) Birds of Chile. Princeton University Press, Princeton.

JIMÉNEZ JE (2007) Ecology of a coastal population of the critically endangered Darwin's fox (Pseudalopex fulvipes) on Chiloé Island, southern Chile. Journal of Zoology 271: 63-77.

JIMENEZ J \& R RAGEOT (1979) Notas sobre la biología del "Monito del Monte", Dromiciops australis Philippi 1893. Anales del Museo de Historia Natural de Valparaíso (Chile) 12: 83-88.

JIMÉNEZ JE \& E MCMAHON (2004) Pseudalopex fulvipes (Martin, 1837). En: Sillero-Zubiri C, M Hoffmann \& DW Macdonald (eds) Canids: Foxes, wolves, jackals and dogs: 50-55. Status survey and conservation action plan, IUCN/SSC Canid Specialist Group, Gland y Oxford, Inglaterra.

JIMÉNEZ JE, M LUCHERINI \& AJ NOVARO (2008) Pseudalopex fulvipes. IUCN red list of threatened species, Version 2009.1. (en línea) URL: http:// www.iucnredlist.org (accedido Marzo 24, 2010).

JOHANSSON ME, C NILSSON \& E NILSSON (1996) Do rivers function as corridors for plant dispersal? Journal of Vegetation Science 7: 593-598.

JOHNSON AW \& JD GOODALL (1967) The birds of Chile and adjacent regions of Argentina, Bolivia and Peru. Platt Establecimientos Gráficos S. A., Buenos Aires.

KILLIAN K (2005) Efectos de los humanos sobre el uso del espacio por los zorros de Darwin (Pseudalopex fulvipes) en Chiloé, Chile. Tesis de Biología, Universidad de Hamburgo, Alemania. 
LARA A, C ECHEVERRÍA \& C DONOSO (2000) Guía de ensayos silviculturales permanentes en los bosques nativos de Chile. World Wide Fund, Instituto de Silvicultura. Universidad Austral de Chile, Valdivia, Chile.

LINDENMAYER DB \& JF FRANKLIN (2002) Conserving forest biodiversity. A comprehensive multiscaled approach. Island Press, Washington, USA.

LOBOS G, A CHARRIER, G CARRASCO \& RE PALMA (2005) Presence of Dromiciops gliroides (Microbiotheria: Microbiotheriidae) in the deciduous forests of central Chile. Mammalian Biology 70: 376-380.

MACDONALD DW \& C SILLERO-ZUBIRI (eds) (2004) Biology and conservation of wild canids. Oxford University Press, Inglaterra.

MARÍN-VIAL P, D GONZÁLEZ-ACUÑA, JL CELISDIEZ, PE CATTAN \& AA GUGLIELMONE (2007) Presence of Ixodes neuquenensis Ringuelet, 1947 (Acari: Ixodidae) on the endangered Neotropical marsupial monito del monte (Dromiciops gliroides Thomas, 1894, Microbiotheria: Microbiotheriidae) at Chiloé Island, Chile. European Journal of Wildlife Research 53: 73-75.

MCPHERSON H (1999) Landscape effects on the distribution of an endemic rhinocryptid, the Ochre-flanked Tapaculo, Eugralla paradoxa, in fragmented south-temperate rainforest. M. Sc. thesis, University of Florida, Gainesville.

MEDEL RG, JE JIMÉNEZ, JL YÁÑEZ, JJ ARMESTO \& FM JAKSIC (1990) Discovery of a continental population of the rare Darwin's fox, Dusicyon fulvipes (Martin, 1837) in Chile. Biological Conservation 51: 71-77.

MERINO S J MORENO, RA VÁSQUEZ, J MARTÍNEZ, I SÁNCHEZ-MONSÁLVEZ et al. (2008) Haematozoa in forest birds from southern Chile: Looking for latitudinal gradients in prevalence and parasite lineage richness. Austral Ecology 33: 329-340.

MERINO S, RA VÁSQUEZ, J MARTÍNEZ, JL CELISDIEZ, L GUTIÉRREZ-JIMENEZ, S IPPI, I SÁNCHEZ-MONSÁLVEZ \& J MARTÍNEZ-DE LA PUENTE (2009) Molecular characterization of an ancient Hepatozoon species parasitizing the "living fossil" marsupial "monito del monte" Dromiciops gliroides from Chile. Biological Journal of the Linnean Society 98: 568-576.

MILLER DS, J ROTTMANN, KJ RAEDECKE \& RD TABER (1983) Endangered mammals of Chile: Status and conservation. Biological Conservation 25: 335-352.

MITTERMEIER RA, GC MITTERMEIER, TM BROOKS, JD PILGRIM, RD KONSTANT, GAB DA FONSECA \& C KORMOS (2003) Wilderness and biodiversity conservation. Proceedings of the National Academy of Sciences USA 100: 10309-10313.

MOORE TR, NT ROULET \& JM WADDINGTON (1998) Uncertainty in predicting the effect of climatic change on the carbon cycling of Canadian peatlands. Climatic Change 40: 229-245.

MORENO J, S MERINO, RA VÁSQUEZ \& JJ ARMESTO (2005) Breeding biology of the thorntailed rayadito (Furnariidae) in south-temperate rainforests of Chile. Condor 107: 69-77.

MORENO J, S MERINO, E LOBATO, MA RODRIGUEZ-GIRONES \& RA VÁSQUEZ (2007)
Sexual dimorphism and parental roles in the thorn-tailed rayadito (Furnariidae). Condor 109: 312-320.

NEPSTAD DC, MOUTINHO P, DIAS-FILHO MB, DAVIDSON E, CARDINOT G et al. (2002) The effects of partial throughfall exclusion on canopy processes, aboveground production, and biogeochemistry of an Amazon forest. Journal of Geophysical Research 107: 8085.

OSGOOD WH (1943) The mammals of Chile. Field Museum of Natural History, Zoological Series 30: $1-268$.

OZANNE CMP, D ANHUF, SL BOULTER, M KELLER, RL KITCHING et al. (2003) Biodiversity meets the atmosphere: A global view of forest canopies. Science 301: 183-186.

PALMA RE \& AE SPOTORNO (1999) Molecular systematics between marsupials based on the rRNA 12S mitochondrial gene: The phylogeny of Didelphimorphia and of the living fossil microbiotheriid Dromiciops gliroides Thomas. Molecular Phylogenetics and Evolution 13: 525-535.

PAPIC C (2000) Regeneración de plántulas arbóreas sobre material leñoso en descomposición en un bosque sucesional de Chiloé, Chile. Tesis de Magíster, Universidad de Chile, Santiago.

PAPIC C \& JJ ARMESTO (2005) Ecología y educación: Hacia una biosfera sustentable. Ambiente y Desarrollo (Chile) 21: 16-19.

PERAKIS SS \& LO HEDIN (2002) Nitrogen loss from unpolluted South American forests mainly via dissolved organic compounds. Nature 415: 416419.

PÉREZ C, M CARMONA, J ARAVENA \& J ARMESTO ( 2004) Successional changes in soil nitrogen availability, no-symbiotic nitrogen fixation and carbon/nitrogen ratios in southern Chilean forest ecosystems. Oecologia 140: 617-625.

PÉREZ CA, MR CARMONA, J FARIÑA \& J ARMESTO (2009) Selective logging of lowland evergreen rainforests in Chiloé Island, Chile. Effects of changing tree species composition on soil nitrogen transformations. Forest Ecology and Management 258: 1660-1668.

QUISPE R, CP VILLAVICENCIO, A CORTES, RA VÁSQUEZ (2009) Inter-population variation in hoarding behaviour in degus, Octodon degus. Ethology 115: 465-474.

RAMOS-JILIBERTO R, AA ALBORNOZ, FS VALDOVINOS, C SMITH-RAMÍREZ, M ARIM, JJ ARMESTO \& PA MARQUET (2009) A network analysis of plant-pollinator interactions in temperate rain forests of Chiloé Island, Chile. Oecologia 160: 697-706.

REID S, IA DÍAZ, JJ ARMESTO \& MF WILLSON (2004) Importance of native bamboo for understory birds in Chilean temperate forests. The Auk 121: 515-525.

REMSEN JV (2003) Family Furnariidae (Ovenbirds). En: del Hoyo J, A Elliot \& DA Christie (eds) Handbook of the birds of the world, Vol. 8: Broadbills to tapaculos: 162-357. Lynx Edicions, Barcelona.

RIVEROS M (1991) Aspectos sobre la biología reproductiva en dos comunidades del sur de Chile, 40 S. Tesis de Doctorado, Facultad de Ciencias, Universidad de Chile, Santiago.

ROZZI R, J SILANDER, JJ ARMESTO, P FEINSINGER \& F MASSARDO (2000) Three levels of 
integrating ecology with the conservation of South American temperate forests: The initiative of the Institute of Ecological Research Chiloé, Chile. Biodiversity and Conservation 9: 11991217.

ROZZI R \& JJ ARMESTO (1996) La propuesta del Instituto de Investigaciones Ecológicas Chiloé. Ambiente y Desarrollo (Chile) 11: 76-81.

ROZZI R, JJ ARMESTO, A CORREA, JC TORRESMURA \& M SALLABERRY (1996) Avifauna of primary temperate forests of uninhabited islands of Chiloe Archipelago, Chile. Revista Chilena De Historia Natural 69: 125-139.

ROZZI R (2003) Guía multi-étnica de aves de los bosques templados de Sudamérica Austral. Fantástico Sur, Santiago, Chile.

SANDERSON J, ME SUNQUIST \& JA IRIARTE (2002) Natural history and landscape-use of guignas (Oncifelis guigna) on Isla Grande de Chiloé, Chile. Journal of Mammalogy 83: 608-613.

SIEVING KE, MF WILLSON \& TL DE SANTO (2000) Defining corridor functions for endemic birds in fragmented south-temperate rainforest. Conservation Biology 14: 1120-1132.

SMITH-RAMÍREZ C (1993) Los picaflores y su recurso floral en el bosque templado de la isla de Chiloé, Chile. Revista Chilena de Historia Natural 66: 6573.

SMITH-RAMÍREZ C \& JJ ARMESTO (1994) Flowering and fruiting patterns in the temperate rainforest of Chiloé, Chile - ecologies and climatic constraints. Journal of Ecology 82: 353-365.

SMITH-RAMÍREZ C \& JJ ARMESTO (2003) Foraging behaviour of bird pollinators on Embothrium coccineum (Proteaceae) trees in forest fragments and pastures in southern Chile. Austral Ecology 28: $53-60$

SMITH-RAMÍREZ C, P MARTÍNEZ, C GONZÁLEZ, M NÚÑEZ \& JJ ARMESTO (2005) Diversity, flower visitation frequency, and generalism of pollinators in temperate rain forests of Chiloé Island, Chile. Botanical Journal of the Linnean Society 147: 399-416.

SMITH-RAMÍREZ C, AE ROVERE, MC NÚÑEZ-ÁVILA \& JJ ARMESTO (2007) Habitat fragmentation and reproductive ecology of Embothrium coccineum, Eucryphia cordifolia and Aextoxicon punctatum in southern temperate rainforests. En: Newton AC (ed) Biodiversity loss \& conservation: 102-119. CABI, Wallingford, UK

TEJO C, I DÍAZ, MR CARMONA, M PEÑA, C PÉREZ \& JJ ARMESTO (2006) Caracterización del suelo epífito presente en individuos emergentes de Eucryphia cordifolia (Eucryphiaceae) en un bosque templado costero de Chiloé, Chile. International Congress on Ecosystem Services in the Neotropics: State of the art and future challenges. FORECOS, Universidad Austral de Chile; November 13-19 2006, Valdivia, Chile. Bosque (Chile) 27: 194

TEWKSBURY JJ, DJ LEVEY, NM HADDAD, S SARGENT, JL ORROCK et al. (2002) Corridors affect plants, animals, and their interactions in fragmented landscapes. Proceedings of the National Academy of Sciences USA 99: 12923-12926.
VAN PELT R (2007) Identifying mature and old forests in western Washington. Washington State Department of Natural Resources, Olympia, WA.

VÁSQUEZ RA \& JA SIMONETTI (1999) Life history traits and sensitivity to landscape change: The case of birds and mammals of mediterranean Chile. Revista Chilena de Historia Natural 72: 517-525.

VILLAGRÁN C (2001) A model for the history of vegetation of the Coastal Range of centralsouthern Chile: Darwin's glacial hypothesis. Revista Chilena de Historia Natural 74: 793-803.

VILLAGRÁN C \& LF HINOJOSA (1997) Historia de los bosques del sur de Sudamérica, II: Análisis fitogeográfico. Revista Chilena de Historia Natural 70: 241-267.

VILLAGRÁN C,P MORENO \& R VILLA (1995) Antecedentes palinológicos acerca de la historias cuaternaria de los bosques chilenos. En: Armesto JJ, C Villagrán \& MTK Arroyo (eds) Ecología de los bosques nativos de Chile: 51-70. Editorial Universitaria, Santiago.

VUILLEUMIER F (1967) Mixed species flocks in Patagonian forests, with remarks on interspecies flock formation. Condor 69: 400-404.

WILLSON MF, JL MORRISON, KE SIEVING, TL DE SANTO, L SANTISTEBAN \& I DÍAZ (2001) Patterns of predation risk and survival of bird nests in a Chilean agricultural landscape. Conservation Biology 15: 447-456.

WILLSON MF, C SMITH-RAMÍREZ, C SABAG \& JF HERNÁNDEZ (1996) Mutualismos entre plantas y animales en bosques templados de Chiloé. En: Armesto JJ, C Villagrán \& MTK Arroyo (eds) Ecología de los bosques nativos de Chile: 251-64. Editorial Universitaria, Santiago.

WILLSON MF, T DE SANTO, C SABAG \& JJ ARMESTO (1994) Avian communities in fragmented south temperate rainforest in Chile. Conservation Biology 8: 508-520.

WILLSON MF \& JJ ARMESTO (1996) The natural history of Chiloé: On Darwin's trail. Revista Chilena de Historia Natural 69: 149-161.

WILLSON MF \& JJ ARMESTO (2003) Efectos de la fragmentación de bosques para las aves de los bosques australes chilenos. Ambiente y Desarrollo (Chile) 19: 54-59.

WILLSON MF (2004) Loss of habitat connectivity hinders pair formation and juvenile dispersal of chucao tapaculos in Chilean rainforest. Condor 106: 166-171.

WILLSON MF, TL DE SANTO, KE SIEVING \& JJ ARMESTO (2005) Success of open-cup nests in south-temperate rainforest fragments in Chile. Boletín Chileno de Ornitología (Chile) 11: 11-17.

WILLSON MF (2006) Chucaos in Chiloé. Boletín Chileno de Ornitología (Chile) 12: 40-44.

WILLSON MF \& GW PENDLETON (2008) Survivorship of chucao tapaculos in fragmented south-temperate rainforest. Boletín Chileno de Ornitología (Chile) 14: 2-7.

YAHNKE CJ, WE JOHNSON, E GEFFEN, D SMITH, F HERTEL et al. (1996) Darwin's fox: A distinct endangered species in a vanishing habitat. Conservation Biology 10: 366-375. 


\section{APENDICE}

\section{La Estación Biológica Senda Darwin}

Actualmente, la EBSD es administrada por la Fundación Senda Darwin -ex Instituto de Investigaciones Ecológicas Chiloé- institución sin fines de lucro dedicada a la integración de la investigación científica con la educación, la aplicación del conocimiento en la toma de decisiones sobre conservación y manejo de bosques templados (Rozzi \& Armesto 1996, Rozzi et al. 2000). Los predios que conforman actualmente la EBSD han sido adquiridos en forma secuencial gracias al aporte de una serie de personas comprometidas con la idea de conservar el bosque nativo (ver agradecimientos). En el año 2003 la EBSD fue reconocida por la Corporación Nacional Forestal (CONAF) como Área Silvestre Protegida Privada, consolidando esta iniciativa de conservación, investigación y educación liderada por ecólogos.

¿Por qué el nombre "Senda Darwin”?

El nombre de la EBSD fue elegido para recordar la visita y recorrido del naturalista inglés Charles Darwin a la zona norte de la Isla de Chiloé, en el año 1834 (Willson \& Armesto 1996). Darwin también es una fuente de inspiración para los investigadores y estudiosos de la Historia Natural, quien fue el precursor de muchas de las ideas centrales de las ciencias ecológicas, y colectó algunas especies emblemáticas de plantas y animales que habitan la zona, como por ejemplo el sapito de Darwin (Rhinoderma darwini), el zorrito chilote (Lycalopex fulvipes) y el "calafate" (Berberis darwini). 